\title{
LOCAL STABILITY ANALYSIS ON LOTKA-VOLTERRA PREDATOR-PREY MODELS WITH PREY REFUGE AND HARVESTING
}

\author{
by \\ Christopher Chow
}

Mathematics and Its Applications, BSc, Ryerson University, 2016

A thesis presented to Ryerson University

in partial fulfilment of the

requirements for the degree of

Master of Science

in the program of

Applied Mathematics

Toronto, Ontario, Canada, 2018

(C) Christopher Chow, 2018 


\section{Author's Declaration}

I hereby declare that I am the sole author of this thesis. This is a true copy of the thesis, including any required final revisions, as accepted by my examiners. I authorize Ryerson University to lend this thesis to other institutions or individuals for the purpose of scholarly research.

I further authorize Ryerson University to reproduce this thesis by photocopying or by other means, in total or in part, at the request of the other institutions or individuals for the purpose of scholarly research.

I understand that my thesis may be made electronically available to the public. 


\title{
LOCAL STABILITY ANALYSIS ON LOTKA-VOLTERRA PREDATOR-PREY MODELS WITH PREY REFUGE AND HARVESTING \\ Christopher Chow \\ Master of Science, 2018 \\ Applied Mathematics \\ Ryerson University
}

\begin{abstract}
We propose a predator-prey model by incorporating a constant harvesting rate into a Lotka-Volterra predator-prey model with prey refuge which was studied recently. All the positive equilibria and the local stability of the proposed model are studied and analyzed by sorting out the intervals of the parameters involved in the model. These intervals of the parameters exhibit the effects on the dynamical behaviors of prey and predators. The emphasis is put on the ranges of the prey refuge constant and harvesting rate. We show that the model has two positive boundary equilibria and one equilibrium. By using the qualitative theory for planar systems, we show that the two positive boundary equilibria can be saddles, saddle-nodes, topological saddles or stable or unstable nodes, and the interior positive equilibrium is locally asymptotically stable. Under suitable restrictions on the parameters, we prove that the positive interior equilibrium is a stable node.
\end{abstract}




\section{ACKNOWLEDGEMENTS}

I would like to thank my supervisor Dr. Kunquan Lan for having been a truly exceptional and dedicated supervisor. I am grateful for the support and guidance Kunquan has given me throughout the last three years of my academic career. He has always inspired me to keep making progress towards furthering my knowledge and research abilities. I would also like to acknowledge my colleagues at Ryerson University, namely Phuc Ngo, Marvin Hoti and Chongming Li, all of whom continue to make contributions in this area of research. They have given me much support in furthering my research, including invaluable advice in overcoming difficulties in my work, as well as spending time to verify and edit some of my results. Finally, I would like to thank the oral examination committee members Pawel Pralat, Silvana Ilie and Dzung Minh Ha for the time and effort in examining my thesis. 


\section{Contents}

Declaration $\quad$ ii

Abstract

Acknowledgements iv

List of Figures $\quad$ vi

1 Introduction 1

1.1 Definitions and Notation . . . . . . . . . . . . . 1

1.2 Local Stability Analysis . . . . . . . . . . . . . . . 2

1.3 Lotka-Volterra Predator-Prey Models . . . . . . . . . . . . 6

1.4 The Proposed Model . . . . . . . . . . . . . . . . 7

2 Qualitative Behaviour of The Equilibrium Points 9

2.1 Equivalent System . . . . . . . . . . . . . . . . 9 9

2.2 Positive Equilibria . . . . . . . . . . . . . . . 12

2.3 Local Stability Analysis . . . . . . . . . . . . . . . 21

2.4 Discussion .......................... 42 
3 Numerical Simulations $\quad 44$

3.1 Example 1..................... . . . 45

3.2 Example $2 \ldots \ldots . \ldots . \ldots . \ldots . \ldots 46$

3.3 Example $3 \ldots \ldots . \ldots . \ldots . \ldots . \ldots 47$

3.4 Example $4 \ldots \ldots \ldots$. . . . . . . . . . . . . . . . . . . . 48

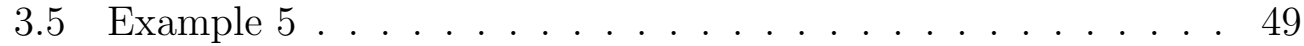

3.6 Example $6 \ldots \ldots \ldots$. . . . . . . . . . . . 50

3.7 Example $7 \ldots \ldots \ldots \ldots \ldots$

$\begin{array}{ll}\text { Bibliography } & 51\end{array}$

$\begin{array}{ll}\text { Index } & 58\end{array}$ 


\section{List of Figures}

3.1 Saddle Boundary Equilibrium (Ex. 1) . . . . . . . . . . 45

3.2 Stable Node Boundary Equilibrium (Ex. 2) . . . . . . . . . . . 46

3.3 Saddle-node Boundary Equilibrium (Ex 3) . . . . . . . . . . 47

3.4 Locally Asymptotically Stable Positive Interior Equilibrium (Ex. 4) . . . . . . . . . . . . . . . . 48

3.5 Locally Asymptotically Stable Positive Interior Equilibrium (Ex. 5) . . . . . . . . . . . . . . . . . 4 49

3.6 Stable Node Positive Interior Equilibrium (Ex. 6) . . . . . . . 50

3.7 Stable Node Positive Interior Equilibrium (Ex. 7) . . . . . . . 51 


\section{Chapter 1}

\section{Introduction}

\subsection{Definitions and Notation}

Consider the following autonomous dynamical system

$$
\left\{\begin{array}{l}
\dot{x}(t):=f(x(t), y(t)), \\
\dot{y}(t):=g(x(t), y(t)) .
\end{array}\right.
$$

For $t \geq 0$, with initial values $(x(0), y(0))=\left(x_{0}, y_{0}\right)$

We use the standard notation for a derivative with respect to time, where $\dot{x}(t)=\frac{d x(t)}{d t}$

Definition 1.1.1. $(x(t), y(t))$ is said to be a solution of (1.1.1) if $x, y \in C^{1}$ and satisfies both equations of (1.1.1). A solution $(x(t), y(t))$ is said to be positive if for all $t \geq 0, x(t), y(t) \geq 0$; a boundary if $x(t) \equiv 0$ or $y(t) \equiv 0$; positive interior if $x(t), y(t)>0$. 
Definition 1.1.2. $\left(x^{*}, y^{*}\right) \in \mathbb{R}^{2}$ is said to be an equilibrium of (1.1.1) if it satisfies $f\left(x^{*}, y^{*}\right)=0$ and $g\left(x^{*}, y^{*}\right)=0$. An equilibrium point $\left(x^{*}, y^{*}\right)$ is said to be positive if $x^{*}, y^{*} \geq 0$; a boundary if $x^{*}=0$ or $y^{*}=0$; positive interior if $x^{*}, y^{*}>0$.

Definition 1.1.3. A map $T: \mathbb{R}^{2} \rightarrow \mathbb{R}^{2}$ defined by $T(x, y)=\left(h_{1}(x, y), h_{2}(x, y)\right)$ is said to be regular if $T$ is one to one and onto, $T$ and $T^{-1}$ are continuous and $|A(x, y)| \neq 0$ on $\mathbb{R}^{2}$.

Definition 1.1.4. If $T$ is regular, then the following transformation

$$
\left\{\begin{array}{l}
x_{1}=h_{1}(x, y), \\
y_{1}=h_{2}(x, y)
\end{array}\right.
$$

is said to be a regular transformation.

If (1.2.1) is changed into another system under suitable regular transformations, then the two systems are said to be equivalent.

\section{$1.2 \quad$ Local Stability Analysis}

In order to analyze the local asymptotic stability near an equilibrium of a system of first order autonomous non-linear scalar differential equations, we can use the method of linearization [2], which is described below.

Consider the autonomous dynamical system of non-linear equations with two variables, $\mathrm{x}$ and $\mathrm{y}$ :

$$
\left\{\begin{array}{l}
\dot{x}(t)=f(x, y), \\
\dot{y}(t)=g(x, y),
\end{array}\right.
$$


Assume the system (1.2.1) has an equilibrium $\left(x^{*}, y^{*}\right)$.

We may expand the functions $f$ and $g$ using the Taylor series centered at $\left(x^{*}, y^{*}\right)$ where $u=x-x^{*}$ and $v=y-y^{*}$ :

$$
\begin{aligned}
& \frac{d u}{d t}=f\left(x^{*}, y^{*}\right)+f_{x}\left(x^{*}, y^{*}\right) u+f_{y}\left(x^{*}, y^{*}\right) v+f_{x x}\left(x^{*}, y^{*}\right) \frac{u^{2}}{2}+f_{x y}\left(x^{*}, y^{*}\right) u v+\ldots \\
& \frac{d v}{d t}=g\left(x^{*}, y^{*}\right)+g_{x}\left(x^{*}, y^{*}\right) u+g_{y}\left(x^{*}, y^{*}\right) v+g_{x x}\left(x^{*}, y^{*}\right) \frac{u^{2}}{2}+g_{x y}\left(x^{*}, y^{*}\right) u v+\ldots
\end{aligned}
$$

Definition 1.2.1. The following system is said to be linearized about the equilibrium $\left(x^{*}, y^{*}\right)$ :

$$
\frac{d \vec{X}}{d t}=A \vec{X}
$$

where $\vec{X}=(u v)^{T}$ and $\mathrm{A}$ is the Jacobian matrix evaluated at the equilibrium $\left(x^{*}, y^{*}\right)$.

We denote by $A(x, y)$ the Jacobian matrix of $f$ and $g$ at $(x, y)$, that is,

$$
A(x, y)=\left(\begin{array}{ll}
\frac{\partial f}{\partial x} & \frac{\partial f}{\partial y} \\
\frac{\partial g}{\partial x} & \frac{\partial g}{\partial y}
\end{array}\right)
$$

and by $|A(x, y)|$ and $\operatorname{tr}(A(x, y))$ its determinant and trace, respectively. 
The eigenvalues of $A(x, y)$ are determined by the roots of the characteristic polynomial:

$$
P(\lambda)=\lambda^{2}-\operatorname{tr}(x, y)+|A(x, y)|
$$

The solutions of a linear system can be studied near the equilibrium $\left(x^{*}, y^{*}\right)$ by the eigenvalues, $\lambda_{1}, \lambda_{2}$, of $A\left(x^{*}, y^{*}\right)$

Definition 1.2.2. An equilibrium of system (1.2.1) is said to be:

(a) a saddle when the eigenvalues of (1.2.2) are real numbers such that $\lambda_{1}<0<\lambda_{2}$.

(b) a node when the eigenvalues are real numbers with the same sign. A node is called a stable when $\lambda_{1,2}<0$. It is called an unstable node when it is not stable; in this case $\lambda_{1,2}>0$.

(c) locally asymptotically stable when eigenvalues are negative or have negative real part.

The following results apply to non-linear planar systems of first order differential equations. The results require that the system may be linearized about an equilibrium, in this case the non-linear system behaves similiarly to a linear system, with some exceptions. The results have been commonly used to study the local stability of biological models based on non-linear systems, for example, in $[11,23,22,13,21,20,16,36]$. We later will use these qualitative theories when discussing local stabilities.

Lemma 1.2.1. [27] Let $\left(u^{*}, v^{*}\right)$ be an equilibrium of (1.2.1). Then the following assertions hold. 
(i) If $\left|A\left(u^{*}, v^{*}\right)\right|<0$, then $\left(u^{*}, v^{*}\right)$ is a saddle.

(ii) If $\left|A\left(u^{*}, v^{*}\right)\right|>0$ and $\left(\operatorname{tr}\left(A\left(u^{*}, v^{*}\right)\right)\right)^{2}-4\left|A\left(u^{*}, v^{*}\right)\right| \geq 0$, then $\left(u^{*}, v^{*}\right)$ is a node. It is stable if $\operatorname{tr}\left(A\left(u^{*}, v^{*}\right)\right)<0$ and unstable if $\operatorname{tr}\left(A\left(u^{*}, v^{*}\right)\right)>0$.

(iii) Assume that $\left|A\left(u^{*}, v^{*}\right)\right|>0$. If $\operatorname{tr}\left(A\left(u^{*}, v^{*}\right)\right)<0$, then $\left(u^{*}, v^{*}\right)$ is locally asymptotically stable; if $\operatorname{tr}\left(A\left(u^{*}, v^{*}\right)\right)=0$, then it is stable and if $\operatorname{tr}\left(A\left(u^{*}, v^{*}\right)\right)>0$, then it is unstable.

Lemma 1.2.2. [20] Let $\left(u^{*}, v^{*}\right)$ be an equilibrium of (1.2.1). Assume that $\left|A\left(u^{*}, v^{*}\right)\right|=0, \operatorname{tr}\left(A\left(u^{*}, v^{*}\right)\right) \neq 0$ and (1.2.1) is equivalent to the following system

$$
\left\{\begin{array}{l}
\dot{u}_{1}=p\left(u_{1}, v_{1}\right) \\
\dot{v}_{1}=\varrho v_{1}+q\left(u_{1}, v_{1}\right)
\end{array}\right.
$$

with an isolated equilibrium $(0,0)$, where $p\left(u_{1}, v_{1}\right)=\sum_{i+j=2, i, j \geq 0}^{\infty} a_{i j} u_{1}^{i} v_{1}^{j}$ and $q\left(u_{1}, v_{1}\right)=\sum_{i+j=2, i, j \geq 0}^{\infty} b_{i j} u_{1}^{i} v_{1}^{j}$ are convergent power series. If $\varrho \neq 0$ and $a_{20} \neq 0$, then $\left(u^{*}, v^{*}\right)$ is a saddle-node.

A function $f: \Omega \subset \mathbb{R}^{2} \rightarrow \mathbb{R}$ is said to be analytic in an open set $\Omega$ if it has a convergent Taylor series in some neighborhood of each point in $\Omega$ (see $[27$, p.69]).

When $\left|A\left(u^{*}, v^{*}\right)\right|=\operatorname{tr}\left(A\left(u^{*}, v^{*}\right)\right)=0$ and $A\left(u^{*}, v^{*}\right) \neq 0$, under suitable regular transformations, (1.2.1) is equivalent to the following form

$$
\left\{\begin{array}{l}
\dot{u}=v \\
\dot{v}=a_{k} u^{k}[1+h(u)]+b_{n} u^{n} v[1+g(u)]+v^{2} R(u, v)
\end{array}\right.
$$


with equilibrium $(0,0)$, where $h, g$ and $R$ are analytic in a neighborhood of $(0,0), h(0)=g(0)=0, k \geq 2, a_{k} \neq 0$ and $n \in \mathbb{N}$.

Lemma 1.2.3 $([1,27])$. Let $\left(u^{*}, v^{*}\right)$ be an equilibrium of $(1.2 .1)$ and $\left|A\left(u^{*}, v^{*}\right)\right|=$ $\operatorname{tr}\left(A\left(u^{*}, v^{*}\right)\right)=0$ and $A\left(u^{*}, v^{*}\right) \neq 0$. If (1.2.1) is equivalent to (1.2.4), $k=2 m+1 \in \mathbb{N}$ and $a_{k}>0$, then $\left(u^{*}, v^{*}\right)$ is a topological saddle.

\subsection{Lotka-Volterra Predator-Prey Models}

Systems of two suitable first order ordinary differential equations can be used to model the interaction between predators and their prey. The dynamical behaviors of the predators and prey can be described by studying the local stability of the planar systems. These dynamical properties provide insight into whether the prey and predators will coexist or suffer from mutual extinction.

One of the classical predator-prey models is the Lotka-Volterra predatorprey model, which is often used to describe the dynamics of biological systems in which two species interact. Since 1925, a variety of predator-prey models have been proposed by introducing functional responses such as HollingTanner predator responses $[4,15,14,21,22,26,28,30,31,32,33,34]$, Beddington-DeAngelis functional responses [3, 5, 10, 20], ratio-dependent functional responses [17, 19, 35], and harvesting rates [13, 18, 20, 22, 35, 36]. The dynamical behaviors of these models have been widely investigated and analyzed. 


\subsection{The Proposed Model}

In 2010, Ma [24] proposed and studied a Lotka-Volterra predator-prey model with a constant prey refuge, which is governed by the following system

$$
\left\{\begin{array}{l}
\dot{x}=r x\left(1-\frac{x}{K}\right)-c(x-R) y, \\
\dot{y}=e(x-R) y-d y
\end{array}\right.
$$

where $x(t)$ and $y(t)$ denote the density of prey and predator populations at time $\mathrm{t}$, respectively, $r$ is the growth rate of the prey, $K$ is the carrying capacity of the prey, $c$ is the per capita rate of predation of the predator (fraction of the prey population eaten per predator), $e$ is a conversion rate of eaten prey into new predator abundance, $d$ is the per capita death rate of the predator and $R$ is the constant prey refuge. The model was further studied and analyzed in $[6,7]$. It was showed in $[6,24]$ that the positive interior equilibrium is globally asymptotically stable and it was proved in [7] that the positive boundary equilibria are saddles for arbitrary prey refuge. Other predator-prey models with constant prey refuges can be found for example, in $[8,12,18,25,29]$.

Harvesting strategies play important roles in the study of optimal management and exploitation renewable resources [9]. A variety of constant or nonconstant harvesting rates have been incorporated into predator-prey models, for example in [13, 18, 20, 22, 35, 36]

In this paper, we incorporate a constant prey harvesting rate, denoted by $h$, into the Lotka-Volterra predator-prey model with prey refuge (1.4.1). This means that prey are being removed from the environment due to some 
external factor, most likely due to humans hunting or poaching the prey population. The proposed model is governed by the following planar system

$$
\left\{\begin{array}{l}
\dot{x}=r x\left(1-\frac{x}{K}\right)-c(x-R) y-h, \\
\dot{y}=e(x-R) y-d y
\end{array}\right.
$$

Following [7], we consider solutions $(x, y)$ of (1.4.2) satisfying $x(t) \geq 0$ and $y(t) \geq 0$ for $t \geq 0$, but unlike the study in $[6,7,24]$, where the authors directly study (1.4.1) without simplification, we use a suitable transformation to change (1.4.2) into an equivalent system and reduce the 7 parameters in (1.4.2) to 4 parameters in the following new system

$$
\left\{\begin{array}{l}
\dot{u}=u(1-u)-(u-\alpha) v-\eta:=f(u, v), \\
\dot{v}=\beta(u-\alpha) v-\gamma v:=g(u, v) .
\end{array}\right.
$$

This model we will be refered to frequently in the following chapters. It will be the main focus of the work done, however all results found for system (1.4.3) will also pertain to the equivalent system (1.4.2) on which the model is based. 


\section{Chapter 2}

\section{Qualitative Behaviour of The Equilibrium Points}

\subsection{Equivalent System}

In this section we provide a method for constructing system (1.4.3), which is equivalent to system (1.4.2). The number of parameters are reduced from 7 to 4 , while at the same time preserving the biological meanings of each parameter. For this reason, we choose to discuss system(1.4.3) instead of system (1.4.2). The method of using suitable transformations to reduce the number of parameters in biological models has been widely used in $[11,13,16,19,20,21,22,23,36]$. We will use system (1.4.3) to study the model using the qualitative theory discussed in the previous chapter, but first we would like to detail the construction of system (1.4.3). 
Theorem 2.1.1. system (1.4.2):

$$
\left\{\begin{array}{l}
\dot{x}=r x\left(1-\frac{x}{K}\right)-c(x-R) y-h \\
\dot{y}=e(x-R) y-d y
\end{array}\right.
$$

is equivalent to system (1.4.3):

$$
\left\{\begin{array}{l}
\dot{u}=u(1-u)-(u-\alpha) v-\eta \\
\dot{v}=\beta(u-\alpha) v-\gamma v
\end{array}\right.
$$

by $\bar{t}=r t, u=x / K$ and $v=c y / r$, where $\alpha=R / K, \beta=e K / r, \gamma=d / r$ and $\eta=h /(K r)$

Proof. Let $\bar{t}=r t, u=x / K$ and $v=c y / r$,

$$
\begin{aligned}
\frac{d u}{d \bar{t}} & =\frac{d u}{d t} \cdot \frac{d t}{d \bar{t}}=\left(\frac{d u}{d x} \cdot \frac{d x}{d t}\right) \frac{d t}{d \bar{t}}=\left(\frac{1}{K} \cdot \frac{d x}{d t}\right) \frac{1}{r} \\
& =\frac{1}{K r}\left[r x\left(1-\frac{x}{K}\right)-c(x-R) y-h\right] \\
& =\frac{1}{K r}\left[r K u\left(1-\frac{K u}{K}\right)-c(K u-R) \frac{r v}{c}-h\right] \\
& =u(1-u)-\left(u-\frac{R}{K}\right) v-\frac{h}{K r} \\
& =u(1-u)-(u-\alpha) v-\eta
\end{aligned}
$$




$$
\begin{aligned}
\frac{d v}{d \bar{t}} & =\frac{d v}{d t} \cdot \frac{d t}{d \bar{t}}=\left(\frac{d v}{d y} \cdot \frac{d y}{d t}\right) \frac{d t}{d \bar{t}}=\left(\frac{c}{r} \cdot \frac{d y}{d t}\right) \frac{1}{r} \\
& =\frac{c}{r^{2}}[e(x-R) y-d y] \\
& =\frac{c}{r^{2}}\left[e(K u-R) \frac{r v}{c}-\frac{d v r}{c}\right] \\
& =\frac{e}{r K}\left(u-\frac{R}{K}\right) v-\frac{d}{r} v \\
& =\beta(u-\alpha) v-\gamma v
\end{aligned}
$$

Let $T: \mathbb{R}^{2} \rightarrow \mathbb{R}^{2}$ be the mapping such that $T$ is defined by the correspondence $T(\boldsymbol{x})=A \boldsymbol{x}$, where $\boldsymbol{x} \in \mathbb{R}^{2}$,

$$
A=\left(\begin{array}{cc}
1 / K & 0 \\
0 & c / r
\end{array}\right) \quad \text { and } \quad|A|=\frac{c}{r K} \neq 0
$$

and $T^{-1}(\boldsymbol{x})=A^{-1} \boldsymbol{x}^{T}$

$$
A^{-1}=\left(\begin{array}{cc}
K & 0 \\
0 & r / c
\end{array}\right) \quad \text { and } \quad|A|=\frac{r K}{c} \neq 0
$$

For any $\boldsymbol{p}, \boldsymbol{q} \in \mathbb{R}^{2}$, where $\boldsymbol{p}=\left(p_{1} p_{2}\right)^{T}$ and $\boldsymbol{q}=\left(q_{1} q_{2}\right)^{T}$ and any scalar $s \in \mathbb{R}$ we have that

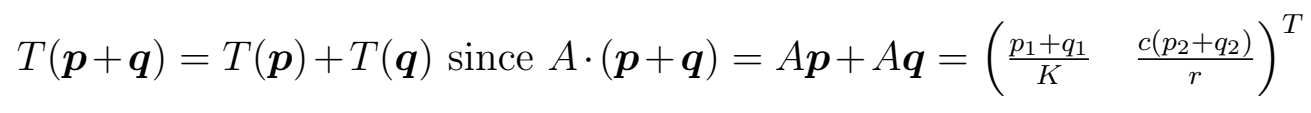

We will show that $T$ is continuous. Let $\epsilon>0$ be given, choose $\delta=\epsilon /\|A\|$. Let $\boldsymbol{x} \in \mathbb{R}^{2}$ and $\boldsymbol{a} \in \mathbb{R}^{2}$ such that $\|\boldsymbol{x}-\boldsymbol{a}\|<\delta$ then

$$
\|T(\boldsymbol{x})-T(\boldsymbol{a})\|=\|T(\boldsymbol{x}-\boldsymbol{a})\|=\|A \cdot(\boldsymbol{x}-\boldsymbol{a})\| \leq\|A\| \cdot\|(\boldsymbol{x}-\boldsymbol{a})\|<\|A\| \delta=\epsilon
$$


Hence, $\|T(\boldsymbol{x})-T(\boldsymbol{a})\|<\epsilon$ when $\|\boldsymbol{x}-\boldsymbol{a}\|<\delta$. Thus $T$ is continuous at $\boldsymbol{a}$ for any $\boldsymbol{a} \in \mathbb{R}^{2}$. Therefore $T$ is continuous on $\mathbb{R}^{2}$. Similarly, $T^{-1}$ can be shown to be continuous. Furthermore, $A \boldsymbol{x}=0$ has only the trivial solution, therefore $T$ is one-to-one. The columns of $A$ span $\mathbb{R}^{2}$, therefore $T$ is onto. By Definition 1.1.3, $T$ is a regular mapping and system (1.4.3) is a regular transformation by Definition 1.1.4, then it is an equivalent to system (1.4.2) with $\bar{t}=r t$.

Corollary 2.1.2. System (1.4.1) is equivalent to the following system

$$
\left\{\begin{array}{l}
\dot{u}=u(1-u)-(u-\alpha) v \\
\dot{v}=\beta(u-\alpha) v-\gamma v
\end{array}\right.
$$

Proof. This is a special case of Theorem (2.1.1), where $h=\eta=0$

Remark 2.1.1. Note that $\alpha=R / K, \beta=e K / r, \gamma=d / r$ and $\eta=h /(K r)$ have same biological meanings as $R, e, d$ and $h$. From (1.4.3).

\subsection{Positive Equilibria}

In this chapter we justify the existence of the equilibria of the model. We find the values of the equilibria with respect to the values of the parameters. Further, we provide the number of equilibria with respect to the values of the parameters.

Following the transformation detailed in the previous chapter, we show that the model can be expressed as system (1.4.3), which are both equiva- 
lent. The latter having less parameters than the former. It is known that under regular transformations, the topological structures of solutions of a planar system near equilibria, including a variety of dynamics like saddles, topological saddles, nodes, saddle-nodes, foci, centers, or cusps, remain unchanged [1]. For these reasons, we consider system (1.4.3) when discussing the equilibria.

Lemma 2.2.1. $(u, v)$ is an equilibrium of system (1.4.3) if and only if $(u, v)$ satisfies

$$
\left\{\begin{array}{l}
u(1-u)-\eta=0 \\
v=0
\end{array}\right.
$$

or

$$
\left\{\begin{array}{l}
u(1-u)-(u-\alpha) v-\eta=0 \\
v \neq 0 \text { and } \quad u=\alpha+\gamma / \beta
\end{array}\right.
$$

Proof. Consider the system (1.4.3), namely:

$$
\left\{\begin{array}{l}
\dot{u}=u(1-u)-(u-\alpha) v-\eta \\
\dot{v}=\beta(u-\alpha) v-\gamma v
\end{array}\right.
$$

By Definition (1.1.2), an equilibrium of system (1.4.3) must satisfy:

$$
\left\{\begin{array}{l}
u(1-u)-(u-\alpha) v-\eta=0 \\
\beta(u-\alpha) v-\gamma v=0
\end{array}\right.
$$


Suppose $v=0$, then the system (2.2.3) becomes:

$$
\left\{\begin{array}{l}
u(1-u)-\eta=0 \\
v=0
\end{array}\right.
$$

Suppose $v \neq 0$, then from the second equation of system (2.2.3) we have:

$$
\begin{aligned}
& \beta(u-\alpha) v-\gamma v=0 \\
\Longrightarrow & \beta(u-\alpha)-\gamma=0 \\
\Longrightarrow & u=\alpha+\gamma / \beta
\end{aligned}
$$

Therefore, the system (2.2.3) becomes:

$$
\left\{\begin{array}{l}
u(1-u)-(u-\alpha) v-\eta=0 \\
v \neq 0 \quad \text { and } \quad u=\alpha+\gamma / \beta
\end{array}\right.
$$


The following results provide the solutions of system (2.2.1) as well as the conditions for which they are positive solutions. It is shown that in order for the system to have positive solutions, the condition $0 \leq \eta \leq 1 / 4$ is required.

Lemma 2.2.2. Let $\beta>0, \gamma>0$ and $\alpha \geq 0$. Then the following assertions hold.

(1) If $\eta>1 / 4$, then (2.2.1) has no real solutions.

(2) If $\eta=1 / 4$, then (2.2.1) has a unique positive solution $(1 / 2,0)$.

(3) If $0 \leq \eta<1 / 4$, then $(2.2 .1)$ has two positive solutions $\left(u_{1}(\eta), 0\right)$ and $\left(u_{2}(\eta), 0\right)$, where

$$
u_{1}(\eta)=1 / 2-\sqrt{1 / 4-\eta} \text { and } u_{2}(\eta)=1 / 2+\sqrt{1 / 4-\eta}
$$

Proof. From the first equation of system (2.2.1) we have:

$$
-u^{2}+u-\eta=0
$$

by solving the quadratic equation we find the solutions:

$$
u_{1}(\eta)=1 / 2-\sqrt{1 / 4-\eta} \quad \text { and } \quad u_{2}(\eta)=1 / 2+\sqrt{1 / 4-\eta}
$$

system $(2.2 .1)$ has two solutions $\left(u_{1}(\eta), 0\right)$ and $\left(u_{2}(\eta), 0\right)$, where $\eta \geq 0$.

(1) Under the condition $\eta>1 / 4$, we find that $\sqrt{1 / 4-\eta}$ is not a real number. In this case, $u_{1}(\eta)$ and $u_{2}(\eta)$ are not real numbers, therefore system (2.2.1) has no real solutions.

(2) Under the condition $\eta=1 / 4$, we have $\left(u_{1}(\eta), 0\right)=\left(u_{2}(\eta), 0\right)=(1 / 2,0)$ is a unique solution of system (2.2.1). 
(3) Under the condition $0 \leq \eta<1 / 4$, we have $1 / 2 \geq \sqrt{1 / 4-\eta}>0$, then

$$
u_{1}(\eta)=1 / 2-\sqrt{1 / 4-\eta} \geq 0 \quad \text { and } \quad u_{2}(\eta)=1 / 2+\sqrt{1 / 4-\eta}>0
$$

Therefore, $\left(u_{1}(\eta), 0\right)$ and $\left(u_{2}(\eta), 0\right)$ are positive solutions of system (2.2.1).

The following result gives the number of positive solutions of system (2.2.2). The range of parameters are determined for which the system has no real solution or a unique solution. We show that in order to ensure existence of the positive interior solution of system (2.2.2), one must restrict the prey refuge and harvesting rate to suitable intervals $\left(0, \alpha_{1}\right)$ and $\left[0, \eta_{0}\right)$, respectively.

Notation: Let $\alpha_{0}=1 / 2-\gamma / \beta, \alpha_{1}=1 / 2+\alpha_{0}$ and

$$
\eta_{0}:=\eta_{0}(\alpha, \beta, \gamma)=\frac{(\gamma+\alpha \beta)(\beta-\gamma-\alpha \beta)}{\beta^{2}}
$$

For $\gamma>0, \beta>0$ and $\alpha \geq 0$,

$$
\eta_{0}=(\alpha+\gamma / \beta)\left(\alpha_{1}-\alpha\right)
$$

and

$$
\eta_{0}=1 / 4-\left(\alpha-\alpha_{0}\right)^{2}
$$

In fact, since $\gamma / \beta=1 / 2-\alpha_{0}$, we have:

$$
\begin{aligned}
\eta_{0} & =(\gamma / \beta+\alpha)[1-(\gamma / \beta+\alpha)]=\left[1 / 2+\left(\alpha-\alpha_{0}\right)\right]\left[1 / 2-\left(\alpha-\alpha_{0}\right)\right] \\
& =1 / 4-\left(\alpha-\alpha_{0}\right)^{2}
\end{aligned}
$$


Lemma 2.2.3. (1) Assume that one of the following conditions holds.

(i) $\beta>0, \gamma>0, \alpha \geq 0$ and $\eta>1 / 4$.

(ii) $0<\beta<\gamma$ and $\alpha \geq 0$ and $0 \leq \eta<1 / 4$.

(iii) $0<\gamma \leq \beta, \alpha>\alpha_{1}$ and $0 \leq \eta<1 / 4$.

(iv) $0<\gamma \leq \beta, 0 \leq \alpha \leq \alpha_{1}, \alpha \neq \alpha_{0}$ if $\alpha_{0}>0$, and $\eta_{0}<\eta<1 / 4$.

Then (2.2.2) has no positive solutions.

(2) If $0<\gamma<\beta, 0 \leq \alpha<\alpha_{1}$ and $0 \leq \eta<\eta_{0}$, then (2.2.2) has a positive interior solution $\left(u^{*}, v^{*}\right)$, where

$$
u^{*}=\alpha+\gamma / \beta \quad \text { and } v^{*}=(\beta / \gamma)\left(\eta_{0}-\eta\right)
$$

Proof. Substituting $u=\alpha+\gamma / \beta=1 / 2+\alpha-\alpha_{0}$ into the first equation of (2.2.2), we have

$$
v=\frac{u(1-u)-\eta}{u-\alpha}=\frac{\left(1 / 2+\alpha-\alpha_{0}\right)\left(1 / 2-\alpha+\alpha_{0}\right)-\eta}{1 / 2-\alpha_{0}}=(\beta / \gamma)\left(\eta_{0}-\eta\right)
$$

and $\eta \neq \eta_{0}$.

(1) It is sufficient to prove $v<0$ under each of the conditions $(i)-(i v)$. If $(i)$ holds, then $\eta>1 / 4$ and by (2.2.6), $\eta_{0} \leq 1 / 4<\eta$. By (2.2.8) that $v<0$. If $(i i)$ or $(i i i)$ holds, then $\alpha>\alpha_{1}$. It follows from $(2.2 .5)$ that $\eta_{0}<0$. By (2.2.8), we have $v<0$. If (iv) holds, by (2.2.5) and (2.2.6), we have $0<\eta_{0}<1 / 4$. Since $\eta_{0}<\eta<1 / 4$, by (2.2.8) we have $v<0$.

(2) Since $\gamma<\beta, 0 \leq \alpha<\alpha_{1}$ and $0 \leq \eta<\alpha_{0}$, by (2.2.5) and (2.2.6), we have $0<\eta_{0}<1 / 4$. Since $0 \leq \eta<\eta_{0}$, by (2.2.8) we have $v>0$. 
Now we present the main result of existence of the equilibria. Below, Theorem 2.2.4 gives the number of equilibria for the entire range of parameters $\alpha, \beta, \gamma$ and $\eta$. We find that there are indeed three potential equilibrium points; two boundary and one interior. The results of this section will be used in the following chapter when discussing the local stability of each equilibrium. Combining Lemmas 2.2.2 and 2.2.3 along with definition 1.1.2, we obtain our main result on the number of positive equilibria of system (1.4.3).

Theorem 2.2.4. (1) If $\beta>0, \gamma>0, \alpha \geq 0$ and $\eta>1 / 4$, then system (1.4.3) has no positive equilibria.

(2) If $\beta>0, \gamma>0, \alpha \geq 0$ and $\eta=1 / 4$, then system (1.4.3) has a unique equilibrium $(1 / 2,0)$.

(3) If one of the following conditions hold:

(i) $0<\beta<\gamma$ and $\alpha \geq 0$ and $0 \leq \eta<1 / 4$.

(ii) $0<\gamma \leq \beta, \alpha>\alpha_{1}$ and $0 \leq \eta<1 / 4$.

(iii) $0<\gamma \leq \beta, 0 \leq \alpha \leq \alpha_{1}, \alpha \neq \alpha_{0}$ if $\alpha_{0}>0$, and $\eta_{0}<\eta<1 / 4$.

then system (1.4.3) has two positive equilibria $\left(u_{1}(\eta), 0\right)$ and $\left(u_{2}(\eta), 0\right)$.

(4) If $0<\gamma<\beta, 0 \leq \alpha<\alpha_{1}$ and $0 \leq \eta<\eta_{0}$, then system (1.4.3) has three equilibria: $\left(u_{1}(\eta), 0\right),\left(u_{2}(\eta), 0\right)$ and $\left(u^{*}, v^{*}\right)$.

Proof. For $(u, v)$ to be an equilibrium of system (1.4.3), by Lemma 2.2.1, it is required to be a solution to either system (2.2.1) or system (2.2.2). Considering this, we prove the claims of the theorem:

(1) Under the conditions $\beta>0, \gamma>0, \alpha \geq 0$ and $\eta>1 / 4$, by Lemma 2.2.2 (1) and Lemma 2.2.3 (1) - (i) we see that system (2.2.3) has no positive 
solutions, therefore system (1.4.3) has no positive equilibria.

(2) Under the conditions $\beta>0, \gamma>0, \alpha \geq 0$ and $\eta=1 / 4$, by Lemma 2.2.2 (2) and Lemma 2.2.3 (1) - (i), system (2.2.3) has a unique positive solution $(1 / 2,0)$, therefore, system $(2.2 .2)$ has a unique equilibrium $(1 / 2,0)$

(3) Under either condition (i), (ii) and (iii), by Lemma 2.2.2 (3) and Lemma 2.2.3(1) - (ii), (1) - (iii) and (1) - (iv), system (2.2.3) has two positive solutions $\left(u_{1}(\eta), 0\right)$ and $\left(u_{2}(\eta), 0\right)$, therefore system (1.4.3) has two positive equilibria $\left(u_{1}(\eta), 0\right)$ and $\left(u_{2}(\eta), 0\right)$.

(4) Under the conditions $0<\gamma<\beta, 0 \leq \alpha<\alpha_{1}$ and $0 \leq \eta<\eta_{0}$, by Lemma 2.2.2 (3) and Lemma 2.2.3(2), system (2.2.3) has three positive solutions $\left(u_{1}(\eta), 0\right)$ and $\left(u_{2}(\eta), 0\right)$ and $\left(u^{*}, v^{*}\right)$, therefore system (1.4.3) has three positive equilibria $\left(u_{1}(\eta), 0\right)$ and $\left(u_{2}(\eta), 0\right)$ and $\left(u^{*}, v^{*}\right)$.

Remark 2.2.1. Theorem 2.2.4 is new and provides the conditions under which the number of positive equilibria of (1.4.3) can be justified. Also, Theorem 2.2.4 (i) and the proof of Lemma 2.2.3 (i) show that if $\eta>1 / 4$, then the predator population becomes extinct. Hence, the harvesting rate $\eta$ must be required to be less than or equal to $1 / 4$ to ensure co-existence of prey and predator populations.

As a special case of Theorem 2.2.4, we obtain the following new result on the number of positive equilibria of (2.1.1).

Corollary 2.2.5. (1) If either $0<\beta<\gamma$ and $\alpha \geq 0$ or $0<\gamma \leq \beta$ and $\alpha>\alpha_{1}$, then (2.1.1) has two positive solutions $(0,0)$ and $(1,0)$. 
(2) If $0<\gamma<\beta$ and $0 \leq \alpha<\alpha_{1}$, then (2.1.1) has three equilibria: $(0,0)$, $(1,0)$ and $\left(u^{*}, v^{*}\right)=\left(\alpha+\gamma / \beta, \beta \eta_{0} / \gamma\right)=\left(\alpha+\gamma / \beta,(1+\alpha \beta / \gamma)\left(\alpha_{1}-\alpha\right)\right)$.

Proof. The result (1) follows from Theorem 2.2.4 (3) with the conditions (ii) and (iii), and the result (2) follows from Theorem 2.2.4 (4).

Remark 2.2.2. The equilibrium $\left(u^{*}, v^{*}\right)$ of (2.1.1) was obtained in [24], also see [6, Thoerem B]. The number of positive equilibria of (2.1.1) was not discussed in $[6,24]$. 


\section{$2.3 \quad$ Local Stability Analysis}

In this chapter, we analyze the local stability of each positive equilibrium of (1.4.3) in order to understand the long term behaviour of the model. Recall the methods discussed in Section 1.2 of Chapter 1, namely from $[1,2,27]$. Now, we use the theoretical results to study phase portraits near each of the positive equilibria of (1.4.3).

For later use within this chapter, we will refer to the following equations which are determined by system (1.4.3) and the Jacobian matrix (1.2.2):

$$
\begin{gathered}
A(u, v)=\left(\begin{array}{cc}
1-2 u-v & -(u-\alpha) \\
\beta v & \beta u-\alpha \beta-\gamma
\end{array}\right), \\
|A(u, v)|=(1-2 u-v)(\beta u-\alpha \beta-\gamma)+\beta v(u-\alpha), \\
\operatorname{tr}(A(u, v))=1-2 u+\beta u-v-\alpha \beta-\gamma .
\end{gathered}
$$

We study now the stability of the boundary equilibria found in the previous chapter, $\left(u_{1}(\eta), 0\right)$ and $(1 / 2,0)$. The three theorems within this section 
analyze each case of boundary equilibrium $(1 / 2,0),\left(u_{1}(\eta), 0\right)$ and $\left(u_{2}(\eta), 0\right)$, respectively.

For convenience, we reiterate the notation introduced in the previous chapter:

$$
\alpha_{0}:=1 / 2-\gamma / \beta, \quad \alpha_{1}:=1 / 2+\alpha_{0} \quad \text { and } \quad \eta_{0}:=1 / 4-\left(\alpha-\alpha_{0}\right)^{2} .
$$

The following theorem contains the results pertaining to the equilibrium $(1 / 2,0)$, which proves the equilibrium to be a saddle-node or a topological saddle under suitable conditions.

Theorem 2.3.1. (1) Assume that one of the following conditions holds,

(i) $0<\beta<2 \gamma$, and $\alpha \geq 0$.

(ii) $0<2 \gamma \leq \beta, \alpha \geq 0$ and $\alpha \neq \alpha_{0}$.

Then $(1 / 2,0)$ is a saddle-node of $(1.4 .3)$ with $\eta=1 / 4$.

(2) If $0<2 \gamma \leq \beta<\infty$ and $\alpha=\alpha_{0}$, then $(1 / 2,0)$ is a topological saddle of (1.4.3) with $\eta=1 / 4$.

Proof. By (2.3.2), and (2.3.3), we have $|A(1 / 2,0)|=0$ and

$$
\operatorname{tr}(A(1 / 2,0))=\beta / 2-\alpha \beta-\gamma=\beta\left(\alpha_{0}-\alpha\right) .
$$

(1) If $0<\beta<2 \gamma$, then $\alpha_{0}<0$, so $(i)$ implies $\operatorname{tr}(A(1 / 2,0))<0$ and $(i i)$ implies $\operatorname{tr}(A(1 / 2,0)) \neq 0$. To apply Lemma 1.2.2, we change the equilibrium $(1 / 2,0)$ to the origin $(0,0)$ by using the change of variables $u_{1}=u-1 / 2$ and 
$v_{1}=v$. Noting that $\eta=1 / 4$, the system (1.4.3) becomes

$$
\left\{\begin{aligned}
\dot{u}_{1} & =\dot{u}=\left(u_{1}+1 / 2\right)\left[1-\left(u_{1}+1 / 2\right)\right]-\left[\left(u_{1}+1 / 2\right)-\alpha\right] v_{1}-1 / 4 \\
& =-u_{1}^{2}-u_{1} v_{1}+\delta_{1} v_{1} \\
\dot{v}_{1} & =\dot{v}=\beta\left(u_{1}+1 / 2-\alpha\right) v_{1}-\gamma v_{1}=\beta u_{1} v_{1}+\delta v_{1}
\end{aligned}\right.
$$

where $\delta=\beta\left(\alpha_{0}-\alpha\right)$ and $\delta_{1}=\alpha-1 / 2$. Let $u_{2}=\delta u_{1}-\delta_{1} v_{1}$ and $v_{2}=v_{1}$, Then the system (2.3.4) becomes

$$
\begin{aligned}
\dot{u}_{2} & =\delta \dot{u}_{1}-\delta_{1} \dot{v}_{1}=\delta\left(-u_{1}^{2}-u_{1} v_{1}+\delta_{1} v_{1}\right)-\delta_{1}\left(\beta u_{1} v_{1}+\delta v_{1}\right) \\
& =-\delta u_{1}^{2}-\left(\delta+\delta_{1} \beta\right) u_{1} v_{1}=-\delta\left(\frac{u_{2}+\delta_{1} v_{2}}{\delta}\right)^{2}-\left(\delta+\delta_{1} \beta\right)\left(\frac{u_{2}+\delta_{1} v_{2}}{\delta}\right) v_{2} \\
& \left.=-(1 / \delta) u_{2}^{2}-(1 / \delta)\left(\delta+2 \delta_{1}+\delta_{1} \beta\right) u_{2} v_{2}+\delta_{1}\left(\delta+\delta_{1}+\delta_{1} \beta\right) v_{2}^{2}\right] \\
\dot{v}_{2} & =\dot{v}_{1}=\beta u_{1} v_{1}+\delta v_{1}=\beta\left(\frac{u_{2}+\delta_{1} v_{2}}{\delta}\right) v_{2}+\delta v_{2}=\delta v_{2}+(\beta / \delta) u_{2} v_{2}+\left(\beta \delta_{1} / \delta\right) v_{2}^{2} .
\end{aligned}
$$

Since $\varrho:=\delta \neq 0$ and $a_{20}:=-1 / \delta \neq 0$, by Lemma $1.2 .2,(1 / 2,0)$ is a saddle-node.

(2) If $0<2 \gamma \leq \beta<\infty$ and $\alpha=\alpha_{0}$, then $\left|A\left(\frac{1}{2}, 0\right)\right|=0, \operatorname{tr}\left(A\left(\frac{1}{2}, 0\right)\right)=0$ and

$$
A(u, v)=\left(\begin{array}{cc}
0 & -\gamma / \beta \\
0 & 0
\end{array}\right) \neq\left(\begin{array}{ll}
0 & 0 \\
0 & 0
\end{array}\right)
$$

Since $\alpha=\alpha_{0}, \delta=0$ and by (2.3.4), we have

$$
\left\{\begin{array}{l}
\dot{u}_{1}=-u_{1}^{2}-u_{1} v_{1}+\delta_{1} v_{1}, \\
\dot{v}_{1}=\beta v_{1} u_{1}
\end{array}\right.
$$

Let $u_{2}=u_{1}, v_{2}=\dot{u}_{1}=-u_{1}^{2}-u_{1} v_{1}+\delta_{1} v_{1}$ and $\delta^{*}=1 / \delta_{1}$. Then

$$
v_{1}=\frac{v_{2}+u_{1}^{2}}{\delta_{1}-u_{1}}=\delta^{*}\left(\frac{v_{2}+u_{2}^{2}}{1-\delta^{*} u_{2}}\right)=\delta^{*}\left(v_{2}+u_{2}^{2}\right)\left[1+\delta^{*} u_{2}+\sum_{n=2}^{\infty}\left(\delta^{*} u_{2}\right)^{n}\right]
$$


and the system (2.3.5) becomes $\dot{u}_{2}=\dot{u}_{1}=v_{2}$,

$$
\begin{aligned}
& \dot{v}_{2}=-2 u_{1} \dot{u}_{1}-\dot{u}_{1} v_{1}-u_{1} \dot{v}_{1}+\delta_{1} \dot{v}_{1}=-2 u_{2} v_{2}-v_{2} v_{1}-\left(u_{2}-\delta_{1}\right) \dot{v}_{1} \\
& =-2 u_{2} v_{2}-v_{2} v_{1}-\left(u_{2}-\delta_{1}\right)\left(\beta v_{1} u_{1}\right)=-2 u_{2} v_{2}-\left(\beta u_{2}^{2}-\beta \delta_{1} u_{2}+v_{2}\right) v_{1} \\
& =-2 u_{2} v_{2}-\left(\beta u_{2}^{2}-\beta \delta_{1} u_{2}+v_{2}\right) \delta^{*}\left(v_{2}+u_{2}^{2}\right)\left[1+\delta^{*} u_{2}+\sum_{n=2}^{\infty}\left(\delta^{*} u_{2}\right)^{n}\right] \\
& =-2 u_{2} v_{2}-\delta^{*}\left(\beta u_{2}^{2}-\beta \delta_{1} u_{2}+v_{2}\right)\left(v_{2}+u_{2}^{2}\right)\left(1+\delta^{*} u_{2}\right)+O_{4}\left(u_{2}, v_{2}\right) \\
& =-2 u_{2} v_{2}-\delta^{*}\left(\beta u_{2}^{2}-\beta \delta_{1} u_{2}+v_{2}\right)\left(v_{2}+\delta^{*} u_{2} v_{2}+u_{2}^{2}+\delta^{*} u_{2}^{3}\right)+O_{4}\left(u_{2}, v_{2}\right) \\
& =-2 u_{2} v_{2}-\delta^{*}\left(\beta u_{2}^{2} v_{2}+\beta \delta^{*} u_{2}^{3} v_{2}+\beta u_{2}^{4}+\beta \delta^{*} u_{2}^{5}-\beta \delta_{1} u_{2} v_{2}-\beta \delta_{1} \delta^{*} u_{2}^{2} v_{2}\right. \\
& \left.-\beta \delta_{1} u_{2}^{3}-\beta \delta_{1} u_{2}^{4}+v_{2}^{2}+\delta^{*} u_{2} v_{2}^{2}+u_{2}^{2} v_{2}+\delta^{*} u_{2}^{3} v_{2}\right)+O_{4}\left(u_{2}, v_{2}\right) \\
& =-2 u_{2} v_{2}-\delta^{*}\left[\left(\beta-\beta \delta_{1} \delta^{*}+1\right) u_{2}^{2} v_{2}+(1+\beta) \delta^{*} u_{2}^{3} v_{2}+\beta\left(1-\delta_{1}\right) u_{2}^{4}+\beta \delta^{*} u_{2}^{5}\right. \\
& \left.-\beta \delta_{1} u_{2} v_{2}-\beta \delta_{1} u_{2}^{3}+v_{2}^{2}+\delta^{*} u_{2} v_{2}^{2}\right]+O_{4}\left(u_{2}, v_{2}\right) \\
& =-2 u_{2} v_{2}+\delta^{*} u_{2}^{2} v_{2}-(1+\beta)\left(\delta^{*}\right)^{2} u_{2}^{3} v_{2}-\beta\left(\delta^{*}-1\right) u_{2}^{4}-\beta\left(\delta^{*}\right)^{2} u_{2}^{5}+\beta u_{2} v_{2} \\
& +\beta u_{2}^{3}-\delta^{*} v_{2}^{2}-\left(\delta^{*}\right)^{2} u_{2} v_{2}^{2}+O_{4}\left(u_{2}, v_{2}\right) \\
& =\beta u_{2}^{3}+(\beta-2) u_{2} v_{2}-\delta^{*} v_{2}^{2}+\delta^{*} u_{2}^{2} v_{2}-(1+\beta)\left(\delta^{*}\right)^{2} u_{2}^{3} v_{2}-\left(\delta^{*}\right)^{2} u_{2} v_{2}^{2} \\
& -\beta\left(\delta^{*}\right)^{2} u_{2}^{5}-\beta\left(\delta^{*}-1\right) u_{2}^{4}+O_{4}\left(u_{2}, v_{2}\right) \\
& =\left[\beta u_{2}^{3}-\beta\left(\delta^{*}\right)^{2} u_{2}^{5}\right]+\left[(\beta-2) u_{2} v_{2}+\delta^{*} u_{2}^{2} v_{2}-(1+\beta)\left(\delta^{*}\right)^{2} u_{2}^{3} v_{2}\right] \\
& -\left[\delta^{*} v_{2}^{2}+\left(\delta^{*}\right)^{2} u_{2} v_{2}^{2}\right]-\beta\left(\delta^{*}-1\right) u_{2}^{4}+O_{4}\left(u_{2}, v_{2}\right) \\
& =\beta u_{2}^{3}\left[1-\left(\delta^{*}-1\right) u_{2}-\left(\delta^{*}\right)^{2} u_{2}^{2}\right]+u_{2} v_{2}\left[(\beta-2)+\delta^{*} u_{2}-(1+\beta)\left(\delta^{*}\right)^{2} u_{2}^{2}\right] \\
& -\delta^{*} v_{2}^{2}\left(1+\delta^{*} u_{2}\right)+O_{4}\left(u_{2}, v_{2}\right) \text {, }
\end{aligned}
$$


where

$$
\begin{aligned}
O_{4}\left(u_{2}, v_{2}\right) & =-\left(\beta u_{2}^{2}-\beta \delta_{1} u_{2}+v_{2}\right) \delta^{*}\left(v_{2}+u_{2}^{2}\right) \sum_{n=2}^{\infty}\left(\delta^{*} u_{2}\right)^{n} \\
& =-\delta^{*}\left(\beta u_{2}^{2} v_{2}+\beta u_{2}^{4}-\beta \delta_{1} u_{2} v_{2}-\beta \delta_{1} u_{2}^{3}+v_{2}^{2}+u_{2}^{2} v_{2}\right) \sum_{n=2}^{\infty}\left(\delta^{*} u_{2}\right)^{n} \\
& =-\delta^{*}\left\{u_{2} v_{2}\left[(1+\beta) u_{2}-\beta \delta_{1}\right]+\beta u_{2}^{3}\left(u_{2}-\delta_{1}\right)+v_{2}^{2}\right\} \sum_{n=2}^{\infty}\left(\delta^{*} u_{2}\right)^{n} \\
& =\left\{u_{2} v_{2}\left[\beta-(1+\beta) \delta^{*} u_{2}\right]+\beta u_{2}^{3}\left(1-\delta^{*} u_{2}\right)-\delta^{*} v_{2}^{2}\right\} \sum_{n=2}^{\infty}\left(\delta^{*} u_{2}\right)^{n}
\end{aligned}
$$

Hence, by (2.3.6) and (2.3.7), we have

$$
\begin{aligned}
\dot{v}_{2} & =\beta u_{2}^{3}\left[1-\left(\delta^{*}-1\right) u_{2}-\left(\delta^{*}\right)^{2} u_{2}^{2}+\left(1-\delta^{*} u_{2}\right) \sum_{n=2}^{\infty}\left(\delta^{*} u_{2}\right)^{n}\right] \\
& +u_{2} v_{2}\left[(\beta-2)+\delta^{*} u_{2}-(1+\beta)\left(\delta^{*}\right)^{2} u_{2}^{2}+\left[\beta-(1+\beta) \delta^{*} u_{2}\right] \sum_{n=2}^{\infty}\left(\delta^{*} u_{2}\right)^{n}\right] \\
& -\delta^{*} v_{2}^{2}\left[1+\delta^{*} u_{2}+\sum_{n=2}^{\infty}\left(\delta^{*} u_{2}\right)^{n}\right] \\
& =\beta u_{2}^{3}\left[1+h\left(u_{2}\right)\right]+(\beta-2) u_{2} v_{2}\left[1+g\left(u_{2}\right)\right]+v_{2}^{2} R\left(u_{2}, v_{2}\right),
\end{aligned}
$$

where

$$
\begin{gathered}
h\left(u_{2}\right)=-\left(\delta^{*}-1\right) u_{2}-\left(\delta^{*}\right)^{2} u_{2}^{2}+\left(1-\delta^{*} u_{2}\right) \sum_{n=2}^{\infty}\left(\delta^{*} u_{2}\right)^{n} \\
g\left(u_{2}\right)=\frac{1}{\beta-2}\left[\delta^{*} u_{2}-(1+\beta)\left(\delta^{*}\right)^{2} u_{2}^{2}+\left[\beta-(1+\beta) \delta^{*} u_{2}\right] \sum_{n=2}^{\infty}\left(\delta^{*} u_{2}\right)^{n}\right]
\end{gathered}
$$

and

$$
R\left(u_{2}, v_{2}\right)=-\delta^{*}-\left(\delta^{*}\right)^{2} u_{2}-\sum_{n=2}^{\infty}\left(\left(\delta^{*}\right)^{2} u_{2}\right)^{n} .
$$

The result follows from Lemma 1.2 .3 with $k=3$ and $n=1$. 
The following theorem contains the results pertaining to the equilibrium $\left(u_{2}(\eta), 0\right)$, which proves the equilibrium to be a saddle, unstable node or a saddle-node under suitable conditions.

Theorem 2.3.2. (1) If one of the following conditions holds,

(i) $0<\beta / 2 \leq \gamma, \alpha \geq 0$ and $0 \leq \eta<\frac{1}{4}$;

(ii) $0<\gamma<\beta / 2$ and $\alpha \geq \alpha_{0}$ and $0 \leq \eta<\frac{1}{4}$;

(iii) $0<\gamma<\beta / 2,0 \leq \alpha<\alpha_{0}$ and $0 \leq \eta<\eta_{0}$

then $\left(u_{1}(\eta), 0\right)$ is a saddle of $(1.4 .3)$.

(2) If $0<\gamma<\beta / 2,0 \leq \alpha<\alpha_{0}$ and $\eta_{0}<\eta<1 / 4$, then $\left(u_{1}(\eta), 0\right)$ is an unstable node of (1.4.3).

(3) If $0<\gamma<\beta / 2,0 \leq \alpha<\alpha_{0}$ and $\eta=\eta_{0}$, then $\left(u_{1}(\eta), 0\right)$ is a saddle-node of (1.4.3).

Proof. By $(2.3 .2)$ with $(u, v)=\left(u_{1}(\eta), 0\right)$ and $u_{1}(\eta)=1 / 2-\sqrt{1 / 4-\eta}$, we have,

$$
\begin{aligned}
\left|A\left(u_{1}(\eta), 0\right)\right| & =\left(1-2 u_{1}(\eta)\right)\left(\beta u_{1}(\eta)-\alpha \beta-\gamma\right) \\
& =2 \sqrt{1 / 4-\eta}[(\beta / 2-\alpha \beta-\gamma)-\beta \sqrt{1 / 4-\eta}] \\
& =2 \beta \sqrt{1 / 4-\eta}\left[\left(\alpha_{0}-\alpha\right)-\sqrt{1 / 4-\eta}\right] .
\end{aligned}
$$

(1) If $(i)$ or $(i i)$ holds, then $\alpha_{0}-\alpha \leq 0$. By (2.3.8), we have $\left|A\left(u_{1}(\eta), 0\right)\right|<$ 0. If $($ iii $)$ holds, then by $\eta<\eta_{0}$, we have $\left(\alpha_{0}-\alpha\right)^{2}<1 / 4-\eta$ and

$$
0 \leq \alpha_{0}-\alpha<\sqrt{1 / 4-\eta}
$$

This, together with (2.3.8), implies $\left|A\left(u_{1}(\eta), 0\right)\right|<0$. 
(2) Since $0 \leq \alpha<\alpha_{0}$ and $\eta_{0}<\eta<1 / 4$, we have $\left(\alpha_{0}-\alpha\right)^{2}>1 / 4-\eta>0$ and

$$
\alpha_{0}-\alpha>\sqrt{1 / 4-\eta}
$$

This, together with (2.3.8), implies $\left|A\left(u_{1}(\eta), 0\right)\right|>0$.

Let

$$
\left.\Delta(u, 0)=[\operatorname{tr}(A(u, 0))]^{2}-4 \mid A(u, 0)\right) \mid \quad \text { for } u \geq 0
$$

Then by $(2.3 .2)$ and (2.3.3) with $(u, v)=(u, 0))$, we have:

$$
\begin{aligned}
\Delta(u, 0) & =[(1-2 u)+(\beta u-(\beta \alpha+\gamma))]^{2}-4(1-2 u)[\beta u-(\beta \alpha+\gamma)] \\
& =(1-2 u)^{2}-2(1-2 u)[\beta u-(\beta \alpha+\gamma)]+(\beta \alpha+\gamma)^{2} \\
& =[(1-2 u)-2(\beta u-(\beta \alpha+\gamma))]^{2} \geq 0
\end{aligned}
$$

It follows that $\Delta\left(u_{1}(\eta), 0\right) \geq 0$.

By (2.3.3) with $(u, v)=\left(u_{1}(\eta), 0\right)$, we have

$$
\begin{aligned}
\operatorname{tr}\left(A\left(u_{1}(\eta), 0\right)\right) & =1-2 u_{1}(\eta)+\beta u_{1}(\eta)-\alpha \beta-\gamma \\
& =2 \sqrt{1 / 4-\eta}+\beta(1 / 2-\sqrt{1 / 4-\eta})-\alpha \beta-\gamma \\
& \left.=2 \sqrt{1 / 4-\eta}+\beta\left(\alpha_{0}-\alpha\right)-\sqrt{1 / 4-\eta}\right)
\end{aligned}
$$

This, together with (2.3.9), implies $\operatorname{tr}\left(A\left(u_{1}(\eta), 0\right)\right)>0$. The result follows from Lemma 1.2.1 (ii).

(3) Since $0 \leq \alpha<\alpha_{0}$ and $\eta=\eta_{0}$, we have $\left(\alpha_{0}-\alpha\right)-\sqrt{1 / 4-\eta}=0$. By (2.3.8) and (2.3.11), $\left|A\left(u_{1}(\eta), 0\right)\right|=0$ and $\operatorname{tr}\left(A\left(u_{1}(\eta), 0\right)\right)>0$.

Let $u_{2}=u-u_{1}(\eta)$ and $v_{2}=v$. Note that $1-2 u_{1}(\eta)=\sqrt{1-4 \eta}$ and 
$u_{1}(\eta)\left[1-u_{1}(\eta)\right]=\eta$. Then the first equation of (1.4.3) becomes

$$
\begin{aligned}
\dot{u}_{2} & =\left(u_{2}+u_{1}(\eta)\right)\left[1-\left(u_{2}+u_{1}(\eta)\right)\right]-\left[\left(u_{2}+u_{1}(\eta)\right)-\alpha\right] v_{2}-\eta \\
& =-u_{2}^{2}-u_{2} v_{2}+\left[1-2 u_{1}(\eta)\right] u_{2}+\left[\alpha-u_{1}(\eta)\right] v_{2}+u_{1}(\eta)\left[1-u_{1}(\eta)\right]-\eta \\
& =-u_{2}^{2}-u_{2} v_{2}+\sqrt{1-4 \eta} u_{2}+\left[\alpha-u_{1}(\eta)\right] v_{2} \\
& =-u_{2}^{2}-u_{2} v_{2}+\delta u_{2}+\delta_{1} v_{2},
\end{aligned}
$$

where $\delta=\sqrt{1-4 \eta}$ and $\delta_{1}=\alpha-u_{1}(\eta)$. By (2.2.6) with $\alpha_{0}>\alpha$, we have

$$
u_{1}(\eta)=u_{1}\left(\eta_{0}\right)=1 / 2-\sqrt{1 / 4-\eta_{0}}=1 / 2-\left(\alpha_{0}-\alpha\right)=\alpha+\gamma / \beta
$$

and $\beta u_{1}(\eta)-\alpha \beta-\gamma=0$. This, together with the second equation of (1.4.3), implies

$$
\dot{v}_{2}=\beta\left(u_{2}+u_{1}(\eta)-\alpha\right) v_{2}-\gamma v_{2}=\beta u_{2} v_{2}+\left[\beta u_{1}(\eta)-\beta \alpha-\gamma\right] v_{2}=\beta u_{2} v_{2}
$$

Let $u_{3}=\delta u_{2}+\delta_{1} v_{2}$ and $v_{3}=v_{2}$. Then $u_{2}=\left(u_{3}-\delta_{1} v_{3}\right) / \delta$ and $(2.3 .12)-(2.3 .13)$ becomes

$$
\begin{aligned}
\dot{u}_{3} & =\delta \dot{u}_{2}+\delta_{1} \dot{v}_{2}=\delta\left(-u_{2}^{2}-u_{2} v_{3}+u_{3}\right)+\delta_{1} \beta u_{2} v_{3} \\
& =-\delta u_{2}^{2}+\left(\beta \delta_{1}-\delta\right) u_{2} v_{3}+\delta u_{3} \\
& =-\frac{1}{\delta}\left[u_{3}-\delta_{1} v_{3}\right]^{2}+\frac{1}{\delta}\left[\left(\beta \delta_{1}-\delta\right)\left(u_{3}-\delta_{1} v_{3}\right) v_{3}\right]+\delta u_{3} \\
& =-\frac{1}{\delta} u_{3}^{2}+\frac{2 \delta_{1}+\beta \delta_{1}-\delta}{\delta} u_{3} v_{3}-\frac{\delta_{1}^{2}+\beta \delta_{1}^{2}-\delta \delta_{1}}{\delta} v_{3}^{2}+\delta u_{3}, \\
\dot{v}_{3} & =\dot{v}_{2}=\frac{\beta}{\delta}\left[u_{3}-\delta_{1} v_{3}\right] v_{3}=-\frac{\beta \delta_{1}}{\delta} v_{3}^{2}+\frac{\beta}{\delta} u_{3} v_{3} .
\end{aligned}
$$


Let $u_{4}=v_{3}$ and $v_{4}=u_{3}$. Then the above system becomes

$$
\begin{aligned}
& \dot{u}_{4}=-\frac{\beta \delta_{1}}{\delta} u_{4}^{2}+\frac{\beta}{\delta} u_{4} v_{4} \\
& \dot{v}_{4}=\delta v_{4}-\frac{1}{\delta} v_{4}^{2}+\frac{2 \delta_{1}+\beta \delta_{1}-\delta}{\delta} u_{4} v_{4}-\frac{\delta_{1}^{2}+\beta \delta_{1}^{2}-\delta \delta_{1}}{\delta} u_{4}^{2} .
\end{aligned}
$$

Since $\varrho:=\delta \neq 0$ and $a_{20}:=-\beta \delta_{1} / \delta \neq 0$, it follows from Lemma 1.2.2 that $\left(u_{1}(\eta), 0\right)$ is a saddle-node.

Corollary 2.3.3. If $\beta>0, \gamma>0$ and $\alpha \geq 0$, then $(0,0)$ is a saddle of (2.1.1).

Proof. By Theorem 2.3.2 (1) with $\eta=0$, we see that the conditions $(i),(i i)$ and ( iii) on $\beta, \gamma$ and $\alpha$ are equivalent to the condition: $\beta>0, \gamma>0, \alpha \geq 0$. The result follows from Theorem 2.3.2 (1) with $\eta=0$.

Remark 2.3.1. Corollary 2.3.3 was proved in [7, Theorem 2.3] by using a different method. By Theorem 2.3.2, we see that the harvesting rate $\eta>0$ affects the dynamical behavior of the model (1.4.3) near the equilibrium $(0,0)$. In particular, when $\eta_{0} \leq \eta<1 / 4$, then Theorem 2.3.2 (2) and (3) are new.

The following theorem contains the results pertaining to the equilibrium $\left(u_{2}(\eta), 0\right)$, which proves the equilibrium to be a saddle, stable node or a saddle-node under suitable conditions.

Theorem 2.3.4. (1) Assume that one of the following conditions holds.

(i) $0<\gamma \leq \beta / 2,0 \leq \alpha \leq \alpha_{0}$ and $0 \leq \eta<1 / 4$.

(ii) $0<\beta / 2<\gamma<\beta, 0 \leq \alpha<\alpha_{1}$ and $0 \leq \eta<\eta_{0}$. 
(iii) $0<\gamma<\beta / 2, \alpha_{0}<\alpha<\alpha_{1}$ and $0 \leq \eta<\eta_{0}$.

Then $\left(u_{2}(\eta), 0\right)$ is a saddle of $(1.4 .3)$.

(2) Assume that one of the following conditions holds.

(i) $0<\beta<\gamma, \alpha \geq 0$ and $0 \leq \eta<1 / 4$.

(ii) $0<\beta / 2<\gamma \leq \beta, 0 \leq \alpha \leq \alpha_{1}$ and $\eta_{0}<\eta<1 / 4$.

(iii) $0<\gamma \leq \beta, \alpha>\alpha_{1}$ and $0 \leq \eta<1 / 4$.

(iv) $0<\gamma \leq \beta / 2, \alpha_{0}<\alpha \leq \alpha_{1}$ and $\eta_{0}<\eta<1 / 4$.

Then $\left(u_{2}(\eta), 0\right)$ is a stable node of $(1.4 .3)$.

(3) Assume that one of the following conditions holds.

(i) If $0<\beta / 2<\gamma \leq \beta, 0 \leq \alpha \leq \alpha_{1}$ and $\eta=\eta_{0}$.

(ii) $0<\gamma \leq \beta / 2, \alpha_{0}<\alpha \leq \alpha_{1}$ and $\eta=\eta_{0}$.

Then $\left(u_{2}(\eta), 0\right)$ is a saddle-node of $(1.4 .3)$.

Proof. By $(2.3 .2)$ with $(u, v)=\left(u_{2}(\eta), 0\right)$ and $u_{2}(\eta)=1 / 2+\sqrt{1 / 4-\eta}$, we have

$$
\begin{aligned}
\left|A\left(u_{2}(\eta), 0\right)\right| & =\left(1-2 u_{2}(\eta)\right)\left(\beta u_{2}(\eta)-\alpha \beta-\gamma\right) \\
& =-2 \sqrt{1 / 4-\eta}[\beta / 2+\beta \sqrt{1 / 4-\eta}-\alpha \beta-\gamma] \\
& =2 \beta \sqrt{1 / 4-\eta}\left[\left(\alpha-\alpha_{0}\right)-\sqrt{1 / 4-\eta}\right] .
\end{aligned}
$$

(1) If $(i)$ holds, then $\alpha-\alpha_{0} \leq 0$ and $\sqrt{1 / 4-\eta}>0$. It follows from (2.3.14) that $\left|A\left(u_{2}(\eta), 0\right)\right|<0$. If $(i i)$ or $(i i i)$, then $\alpha-\alpha_{0}>0$. Since $0 \leq \eta<\eta_{0}$, by (2.2.6) we have $0 \leq \eta<\eta_{0}=1 / 4-\left(\alpha-\alpha_{0}\right)^{2}$ and $\left(\alpha-\alpha_{0}\right)^{2}<1 / 4-\eta$. This implies $0<\alpha-\alpha_{0}<\sqrt{1 / 4-\eta}$. By (2.3.14), we have $\left|A\left(u_{2}(\eta), 0\right)\right|<0$. By Lemma 1.2.1 $(1),\left(u_{2}(\eta), 0\right)$ is a saddle of (1.4.3). 
(2) We first prove $\left|A\left(u_{2}(\eta), 0\right)\right|>0$. By $(2.3 .14)$, we see that $\left|A\left(u_{2}(\eta), 0\right)\right|>$ 0 if and only if

$$
\alpha>\alpha_{0} \quad \text { and } \eta>\eta_{0}
$$

Hence, it is sufficient to show that under each of the conditions $(i)-(i v)$, (2.3.15) holds. In fact, $(i)$ implies $\alpha_{0}<\alpha_{1}<0 \leq \alpha$ and $\eta_{0}<0 \leq \eta$; $(i i)$ implies $\alpha_{0}<0 \leq \alpha$ and $0 \leq \eta_{0}<\eta$; (iii) implies $\alpha_{0}<0<\alpha_{1}<\alpha$ and $\eta_{0}<0 \leq \eta$ and $(i v)$ implies $0 \leq \alpha_{0}<\alpha<\alpha_{1}$ and $0 \leq \eta_{0}<\eta$. Hence, we have proved $\left|A\left(u_{2}(\eta), 0\right)\right|>0$. By $(2.3 .10), \Delta\left(u_{2}(\eta), 0\right) \geq 0$. By $(2.3 .3)$ with $(u, v)=\left(u_{2}(\eta), 0\right)$ and $(2.3 .14)$, we have

$$
\begin{aligned}
\operatorname{tr}\left(A\left(u_{2}(\eta), 0\right)\right) & =1-2 u_{2}(\eta)+\beta u_{2}(\eta)-\alpha \beta-\gamma \\
& =-2 \sqrt{1 / 4-\eta}+\beta / 2+\beta \sqrt{1 / 4-\eta}-\alpha \beta-\gamma \\
& =-2 \sqrt{1 / 4-\eta}-\beta\left(\alpha-\alpha_{0}-\sqrt{1 / 4-\eta}\right) \\
& =-2 \sqrt{1 / 4-\eta}-\frac{\left|A\left(u_{2}(\eta), 0\right)\right|}{2 \sqrt{1 / 4-\eta}}<0
\end{aligned}
$$

The result follows from Lemma 1.2.1 (ii).

(3) Since $\eta_{0}=1 / 4-\left(\alpha-\alpha_{0}\right)^{2}$, under each of the condition $(i)$ and (ii), by (2.3.14) and (2.3.16) with $\eta=\eta_{0}$, we have $\left|A\left(u_{2}(\eta), 0\right)\right|=0$ and $\operatorname{tr}\left(A\left(u_{2}(\eta), 0\right)\right)<0$.

Let $u_{3}=u-u_{2}(\eta)$ and $v_{3}=v$. Note that $2 u_{2}(\eta)-1=\sqrt{1-4 \eta}$ and 
$u_{2}(\eta)\left[1-u_{2}(\eta)\right]=\eta$. Then the first equation of (1.4.3) becomes

$$
\begin{aligned}
\dot{u}_{3} & =\left(u_{3}+u_{2}(\eta)\right)\left[1-\left(u_{3}+u_{2}(\eta)\right)\right]-\left[\left(u_{3}+u_{2}(\eta)\right)-\alpha\right] v_{3}-\eta \\
& =-u_{3}^{2}-u_{3} v_{3}+\left[1-2 u_{2}(\eta)\right] u_{3}+\left[\alpha-u_{2}(\eta)\right] v_{3}+u_{2}(\eta)\left[1-u_{2}(\eta)\right]-\eta \\
& =-u_{3}^{2}-u_{3} v_{3}+\sqrt{1-4 \eta} u_{3}+\left[\alpha-u_{2}(\eta)\right] v_{3} \\
& =-u_{3}^{2}-u_{3} v_{3}+\delta u_{3}+\delta_{1} v_{3}
\end{aligned}
$$

where $\delta=\sqrt{1-4 \eta}$ and $\delta_{1}=\alpha-u_{2}(\eta)$. Under each of the conditions $(i)$ and (ii), we have $\alpha-\alpha_{0}>0$. By (2.2.6) with $\eta=\eta_{0}$, we have

$$
u_{2}(\eta)=u_{2}\left(\eta_{0}\right)=1 / 2+\sqrt{1 / 4-\eta_{0}}=1 / 2+\alpha-\alpha_{0}=\alpha+\gamma / \beta
$$

This implies $\beta u_{2}(\eta)=\beta u_{2}\left(\eta_{0}\right)-\alpha \beta-\gamma=0$. This, together with the second equation of (1.4.3), implies

$$
\dot{v}_{3}=\beta\left(u_{3}+u_{2}(\eta)-\alpha\right) v_{3}-\gamma v_{3}=\beta u_{3} v_{3}+\left[\beta u_{2}(\eta)-\alpha \beta-\gamma\right] v_{3}=\beta u_{3} v_{3} .
$$

Let $u_{4}=\delta u_{3}+\delta_{1} v_{3}$, and $v_{4}=v_{3}$. Then $u_{3}=\left(u_{4}-\delta_{1} v_{4}\right) / \delta$ and (2.3.17)(2.3.18) becomes

$$
\begin{aligned}
\dot{u}_{4} & =\delta \dot{u}_{3}+\delta_{1} \dot{v}_{3}=\delta\left(-u_{3}^{2}-u_{3} v_{3}+\delta u_{3}+\delta_{1} v_{3}\right)+\delta_{1} \beta u_{3} v_{4} \\
& =\delta\left(-u_{3}^{2}-u_{3} v_{4}+u_{4}\right)+\delta_{1} \beta u_{3} v_{4}=-\delta u_{3}^{2}+\left(\beta \delta_{1}-\delta\right) u_{3} v_{4}+\delta u_{4} \\
& =-\frac{1}{\delta}\left[u_{4}-\delta_{1} v_{4}\right]^{2}+\frac{1}{\delta}\left[\left(\beta \delta_{1}-\delta\right)\left(u_{4}-\delta_{1} v_{4}\right) v_{4}\right]+\delta u_{4} \\
& =-\frac{1}{\delta} u_{4}^{2}+\frac{2 \delta_{1}+\beta \delta_{1}-\delta}{\delta} u_{4} v_{4}-\frac{\delta_{1}\left[(1+\beta) \delta_{1}-\delta\right]}{\delta} v_{4}^{2}+\delta u_{4}, \\
\dot{v}_{4} & =\dot{v}_{3}=\frac{\beta}{\delta}\left[u_{4}-\delta_{1} v_{4}\right] v_{4}=-\frac{\beta \delta_{1}}{\delta} v_{4}^{2}+\frac{\beta}{\delta} u_{4} v_{4} .
\end{aligned}
$$


Let $u_{5}=v_{4}$ and $v_{5}=u_{4}$. Then the above system becomes

$$
\begin{aligned}
& \dot{u}_{5}=-\frac{\beta \delta_{1}}{\delta} u_{5}^{2}+\frac{\beta}{\delta} u_{5} v_{5}, \\
& \dot{v}_{5}=\delta v_{5}-\frac{1}{\delta} v_{5}^{2}+\frac{2 \delta_{1}+\beta \delta_{1}-\delta}{\delta} u_{5} v_{5}-\frac{\delta_{1}\left[(1+\beta) \delta_{1}-\delta\right]}{\delta} u_{5}^{2} .
\end{aligned}
$$

Since $\varrho:=\delta \neq 0$ and $a_{20}:=-\beta \delta_{1} / \delta \neq 0$, it follows from Lemma 1.2.2 that $\left(u_{2}(\eta), 0\right)$ is a saddle-node of $(1.4 .3)$.

Corollary 2.3.5. (1) If either $0<\gamma<\beta$ with $\gamma \neq \beta / 2$ and $0 \leq \alpha<\alpha_{1}$ or $0<\gamma=\beta / 2$ and $\alpha=0$, then $(1,0)$ is a saddle of $(2.1 .1)$.

(2) If either $0<\beta<\gamma$ and $\alpha \geq 0$ or $0<\gamma \leq \beta$ and $\alpha>\alpha_{1}$, then $(1,0)$ is a stable node of (2.1.1).

(3) If $0<\gamma \leq \beta$ and $\alpha=\alpha_{1}$, then $(1,0)$ is a saddle-node of (2.1.1).

Proof. (1) The conditions in (1) are equivalent to the conditions $(i),(i i)$ and (iii) in Theorem 2.3.4 (1) with $\eta=0$. The result (1) follows from Theorem 2.3 .4 (1) with $\eta=0$. The result (2) follows from (i) and (iii) of Theorem 2.3.4 (2) with $\eta=0$ and the result (3) follows from Theorem 2.3.4 (2).

Remark 2.3.2. Corollary 2.3.5 shows that the equilibrium $(1,0)$ can be a saddle, a stable node or a saddle-node for suitable choice of the refuge constant $\alpha$. However, [7, Theorem 2.3] shows that $(1,0)$ only is a saddle for all refuge values, $\alpha \geq 0$, by using a different method. Figure 1 given in section 4 shows that $(1,0)$ can be a stable node or a saddle-node.

We now study the phase portraits near the positive interior equilibrium $\left(u^{*}, v^{*}\right)$ of system (1.4.3). 
For convenience, we provide a summary of the the notation used in this section:

$$
\begin{gathered}
\alpha_{0}:=1 / 2-\gamma / \beta, \quad \alpha_{1}:=1 / 2+\alpha_{0}, \quad \alpha_{2}(\beta, \gamma):=\frac{1}{2}-\frac{\sqrt{\omega(\beta, \gamma)}}{\sqrt{12} \beta}, \\
\alpha_{3}(\beta, \gamma):=\frac{1}{2}+\frac{\sqrt{\omega(\beta, \gamma)}}{\sqrt{12} \beta}, \quad \eta_{0}:=1 / 4-\left(\alpha-\alpha_{0}\right)^{2} \\
\eta_{1}:=\gamma^{2} / \beta^{2}+1 / 4-(\alpha-1 / 2)^{2}, \quad \Delta(\alpha, \eta):=\left[\operatorname{tr}\left(A\left(u^{*}, v^{*}\right)\right)\right]^{2}-4\left|A\left(u^{*}, v^{*}\right)\right| \\
\omega(\beta, \gamma):=\beta^{2}-8 \beta \gamma^{2}+4 \gamma^{2}, \quad \varrho:=\max \left\{\frac{\beta}{\sqrt{2(1+\beta)}}, \frac{4 \beta^{2}}{1+4 \beta}\right\}
\end{gathered}
$$

The following theorem proves that $\left(u^{*}, v^{*}\right)$ is stable, unstable or locally symptotically stable.

Theorem 2.3.6. (1) Assume that one of the following conditions holds.

(i) $0<\gamma<\beta / 2,0 \leq \alpha<\alpha_{0}$ and $0 \leq \eta<\eta_{1}$, where $\eta_{1}=\gamma^{2} / \beta^{2}+\alpha(1-\alpha)$.

(ii) $0<\gamma<\beta, \max \left\{0, \alpha_{0}\right\} \leq \alpha<\alpha_{1}$ and $0 \leq \eta<\eta_{0}$.

Then $\left(u^{*}, v^{*}\right)$ is a locally asymptotically stable.

(2) Assume that $0<\gamma<\beta / 2$ and $0 \leq \alpha<\alpha_{0}$. If $\eta=\eta_{1}$, then $\left(u^{*}, v^{*}\right)$ is stable, and if $\eta_{1}<\eta<\eta_{0}$, then $\left(u^{*}, v^{*}\right)$ is unstable.

Proof. By (2.2.7) we have $\beta u^{*}-\alpha \beta-\gamma=0$ and (2.3.1) becomes

$$
A\left(u^{*}, v^{*}\right)=\left(\begin{array}{cc}
1-2 u^{*}-v^{*} & -\left(u^{*}-\alpha\right) \\
\beta v^{*} & 0
\end{array}\right)
$$

We first prove that if $0<\gamma<\beta, 0 \leq \alpha<\alpha_{1}$ and $0 \leq \eta<\eta_{0}$, then

$$
\left|A\left(u^{*}, v^{*}\right)\right|=\beta\left(\eta_{0}-\eta\right)>0 \text {. }
$$


Indeed,

$$
\begin{aligned}
\left|A\left(u^{*}, v^{*}\right)\right| & =\beta v^{*}\left(u^{*}-\alpha\right)=\beta(\beta / \gamma)\left(\eta_{0}-\eta\right)(\gamma / \beta) \\
& =\beta\left(\eta_{0}-\eta\right)=\beta\left(\eta_{0}-\eta\right)>0 .
\end{aligned}
$$

(1) If $0 \leq \alpha<\alpha_{0}$, then $\eta_{1}>0$ since

$\eta_{1}=\gamma^{2} / \beta^{2}+1 / 4-(\alpha-1 / 2)^{2}=\left(\frac{\sqrt{\beta^{2}+4 \gamma^{2}}+\beta}{2 \beta}-\alpha\right)\left(\frac{\sqrt{\beta^{2}+4 \gamma^{2}}-\beta}{2 \beta}+\alpha\right)$.

By (2.2.6), we have

$$
\begin{aligned}
\eta_{0}-\eta_{1} & =1 / 4-\left(\alpha-\alpha_{0}\right)^{2}-\gamma^{2} / \beta^{2}-\alpha(1-\alpha)=\left(\alpha_{0}-\alpha\right)\left(1-2 \alpha_{0}\right) \\
& =(2 \gamma / \beta)\left(\alpha_{0}-\alpha\right)
\end{aligned}
$$

By (2.3.19) and Lemma 2.2.3 (2) we have

$$
\begin{aligned}
\operatorname{tr}\left(A\left(u^{*}, v^{*}\right)\right)= & 1-2 u^{*}-v^{*} \\
= & 1-2(\alpha+\gamma / \beta)-(\beta / \gamma)[(\alpha+\gamma / \beta)(1-\gamma / \beta-\alpha)-\eta] \\
= & (\beta / \gamma)[\gamma / \beta-2(\gamma / \beta)(\alpha+\gamma / \beta)-(\alpha+\gamma / \beta)(1-\alpha-\gamma / \beta)+\eta] \\
= & (\beta / \gamma)\left[\gamma / \beta-2 \alpha \gamma / \beta-2 \gamma^{2} / \beta^{2}+(\gamma / \beta)(\alpha+\gamma / \beta)-\alpha(1-\alpha)\right. \\
& -(\gamma / \beta)(1-\alpha)+\eta] \\
= & (\beta / \gamma)\left[\eta-\gamma^{2} / \beta^{2}-\alpha(1-\alpha)\right]=(\beta / \gamma)\left(\eta-\eta_{1}\right) .
\end{aligned}
$$

If $\max \left\{0, \alpha_{0}\right\} \leq \alpha<\alpha_{1}$, then $\alpha_{0}-\alpha \leq 0$ and by (2.3.21), we have $\eta_{0} \leq \eta_{1}$. It follows from $0 \leq \eta<\eta_{0}$ that $\eta-\eta_{1}<0$. By $(2.3 .22), \operatorname{tr}\left(A\left(u^{*}, v^{*}\right)\right)<0$. If $0 \leq \alpha<\alpha_{0}$, then

$$
\gamma^{2} / \beta^{2}<\eta_{1}<\gamma^{2} / \beta^{2}+\alpha_{0}\left(1-\alpha_{0}\right)=1 / 4
$$


By $(2.3 .21), \eta_{1}<\eta_{0}$ and by $(2.3 .22), \operatorname{tr}\left(A\left(u^{*}, v^{*}\right)\right)<0$. It follows from Lemma 1.2.1 $(i v)$ that $\left(u^{*}, v^{*}\right)$ is locally asymptotically stable.

The result (2) follow from (2.3.22) and Lemma 1.2.1 (iv).

Corollary 2.3.7. If $0<\gamma<\beta$ and $0 \leq \alpha<\alpha_{1}$, then $\left(u^{*}, v^{*}\right)$ is a locally asymptotically stable equilibrium of (2.1.1).

Proof. The conditions $(i)$ and $(i i)$ with $\eta=0$ of Theorem 2.3.6 (1) are equivalent to $(i) 0<\beta / 2<\gamma<\beta$ and $0 \leq \alpha<\alpha_{1}$; (ii) $0<\gamma \leq \beta / 2$, $\alpha_{0} \leq \alpha<\alpha_{1}$ and $\left(\right.$ iii) $0<\gamma<\beta / 2,0 \leq \alpha<\alpha_{0}$, which are equivalent to $0<\gamma<\beta$ and $0 \leq \alpha<\alpha_{1}$. The result follows from Theorem 2.3.6 (1) with $\eta=0$.

Remark 2.3.3. Theorem 2.3.6 (1) provides the conditions on the prey refuge and harvesting rates under which the interior equilibrium $\left(u^{*}, v^{*}\right)$ of $(1.4 .3)$ is locally asymptotically stable. Theorem 2.3.6 (1) with $\eta=0$, that is, Corollary 2.3.7 improves [7, Lemma 2.2 and Theorem 2.5] with $m=1$, where it is proved that $\left(u^{*}, v^{*}\right)$ is locally asymptotically stable and globally asymptotically stable, respectively. Theorem 2.3.6 (2) and (3) are new results.

In the following, we show that under suitable conditions on the parameters, $\left(u^{*}, v^{*}\right)$ is a stable node of (1.4.3).

Let

$$
\Delta(\alpha, \eta):=\left[\operatorname{tr}\left(A\left(u^{*}, v^{*}\right)\right)\right]^{2}-4\left|A\left(u^{*}, v^{*}\right)\right|
$$

and for $0<\gamma<\beta$, let

$$
\omega(\beta, \gamma)=\beta^{2}-8 \beta \gamma^{2}+4 \gamma^{2}
$$


Lemma 2.3.8. (1) If $\frac{4 \beta^{2}}{1+4 \beta} \leq \gamma<\beta$, then $\Delta(0,0) \geq 0$.

(2) If $\beta>0$ and $\gamma>\max \{0,(2 \beta-1) / 4\}$, then $\Delta_{\alpha}^{\prime}(0,0)>0$.

(3) If $0<\gamma<\beta$ and $0 \leq \alpha<\alpha_{1}$, then

$$
\Delta_{\alpha}^{\prime \prime}(\alpha, 0)=\frac{12 \beta^{2}}{\gamma^{2}}\left[\left(\alpha-\frac{1}{2}\right)^{2}-\frac{\omega(\beta, \gamma)}{12 \beta^{2}}\right]
$$

Proof. By (2.3.20) and (2.3.22), we have

$$
\Delta(\alpha, \eta)=\frac{\beta^{2}}{\gamma^{2}}\left(\eta-\eta_{1}\right)^{2}-4 \beta\left(\eta_{0}-\eta\right)
$$

(1) Since $\Delta(0,0)=\gamma^{2} / \beta^{2}-4 \beta\left[1 / 4-\alpha_{0}^{2}\right]$ and $\alpha_{0}=1 / 2-\gamma / \beta$, we have

$$
\begin{aligned}
\Delta(0,0) & =\gamma^{2} / \beta^{2}-\beta+4 \beta(1 / 2-\gamma / \beta)^{2}=\gamma^{2} / \beta^{2}-4 \gamma+4 \gamma^{2} / \beta^{2} \\
& =\frac{\gamma}{\beta^{2}}\left(\gamma-4 \beta^{2}+4 \beta \gamma\right)=\frac{\gamma(1+4 \beta))}{\beta^{2}}\left(\gamma-\frac{4 \beta^{2}}{1+4 \beta}\right)
\end{aligned}
$$

from which we see that the result holds.

(2) By (2.3.26) with $\eta=0$ and (2.2.6), we have for $\alpha \geq 0$,

$$
\Delta(\alpha, 0)=\frac{\beta^{2}}{\gamma^{2}} \eta_{1}^{2}-4 \beta \eta_{0}=\frac{\beta^{2}}{\gamma^{2}}\left[\frac{\gamma^{2}}{\beta^{2}}-\alpha(\alpha-1)\right]^{2}-4 \beta\left[1 / 4-\left(\alpha-\alpha_{0}\right)^{2}\right]
$$

and

$$
\Delta_{\alpha}^{\prime}(\alpha, 0)=\frac{2 \beta^{2}}{\gamma^{2}}\left[\frac{\gamma^{2}}{\beta^{2}}-\alpha(\alpha-1)\right](1-2 \alpha)+8 \beta\left(\alpha-\alpha_{0}\right)
$$

It follows that

$$
\begin{aligned}
\Delta_{\alpha}^{\prime}(0,0) & =\frac{2 \beta^{2}}{\gamma^{2}}\left[\frac{\gamma^{2}}{\beta^{2}}-0\right](1-0)+8 \beta\left(0-\alpha_{0}\right)=2-8 \beta \alpha_{0}=2-8 \beta(1 / 2-\gamma / \beta) \\
& =8\left[\gamma-\frac{2 \beta-1}{4}\right]>0
\end{aligned}
$$


(3) By (2.3.28), we have for $\alpha \geq 0$,

$$
\begin{aligned}
\Delta^{\prime \prime}(\alpha, 0) & =\frac{2 \beta^{2}}{\gamma^{2}}(2 \alpha-1)^{2}-\frac{4}{\gamma^{2}}\left[\gamma^{2}-\beta^{2} \alpha(\alpha-1)\right]+8 \beta \\
& =\frac{12 \beta^{2}}{\gamma^{2}}\left[\left(\alpha-\frac{1}{2}\right)^{2}-\frac{\beta^{2}-8 \beta \gamma^{2}+4 \gamma^{2}}{12 \beta^{2}}\right]=\frac{12 \beta^{2}}{\gamma^{2}}\left[\left(\alpha-\frac{1}{2}\right)^{2}-\frac{\omega(\beta, \gamma)}{12 \beta^{2}}\right]
\end{aligned}
$$

The result (3) holds.

Lemma 2.3.9. (1) If either $0<\beta \leq 5 / 8$ and $0<\gamma<\beta$ or $\beta>5 / 8$ and $0<\gamma<\frac{\beta}{2 \sqrt{2 \beta-1}}$, then $\omega(\beta, \gamma)>0$.

(2) If $\beta>5 / 8$ and $\frac{\beta}{2 \sqrt{2 \beta-1}} \leq \gamma<\beta$, then $\omega(\beta, \gamma) \leq 0$.

Proof. (1) If $0<\gamma<\beta \leq 1 / 2$, then by (2.3.24), $\omega(\beta, \gamma)=\beta^{2}+4 \gamma^{2}(1-2 \beta)>$ 0 . If $\beta>1 / 2$, then

$$
\omega(\beta, \gamma)=4(2 \beta-1)\left(\frac{\beta}{2 \sqrt{2 \beta-1}}+\gamma\right)\left(\frac{\beta}{2 \sqrt{2 \beta-1}}-\gamma\right) .
$$

It is easy to verify that if $1 / 2<\beta \leq 5 / 8$, then $\beta \leq \frac{\beta}{2 \sqrt{2 \beta-1}}$. If $1 / 2<\beta \leq 5 / 8$ and $0<\gamma<\beta$ or $\beta>5 / 8$ and $0<\gamma<\frac{\beta}{2 \sqrt{2 \beta-1}}$, then by(2.3.29), $\omega(\beta, \gamma)>0$.

(2) Since $\beta>5 / 8$ and $\frac{\beta}{2 \sqrt{2 \beta-1}} \leq \gamma<\beta$, by (2.3.29) we have $\omega(\beta, \gamma) \leq$ 0.

Theorem 2.3.10. (1) If $\beta>5 / 8, \max \left\{\frac{4 \beta^{2}}{1+4 \beta}, \frac{\beta}{2 \sqrt{2 \beta-1}}\right\} \leq \gamma<\beta$ and $\max \left\{0, \alpha_{0}\right\} \leq$ $\alpha<\alpha_{1}$, then there exists $\eta_{1}^{*} \in\left(0, \eta_{0}\right)$ such that $\left(u^{*}, v^{*}\right)$ is a stable node of (1.4.3) for $\eta \in\left[0, \eta_{1}^{*}\right]$.

(2) If $\beta \geq 1, \frac{\beta}{2 \sqrt{2 \beta-1}} \leq \gamma \leq \frac{\beta}{2}$ and $0 \leq \alpha<\alpha_{0}$, then there exists $\eta_{2}^{*} \in\left(0, \eta_{1}\right)$ such that $\left(u^{*}, v^{*}\right)$ is a stable node of $(1.4 .3)$ for $\eta \in\left[0, \eta_{2}^{*}\right]$.

Proof. (1) Since $\beta>5 / 8, \max \left\{\frac{4 \beta^{2}}{1+4 \beta}, \frac{\beta}{2 \sqrt{2 \beta-1}}\right\} \leq \gamma<\beta$, by Lemma 2.3 .9 (2), $\omega(\beta, \gamma) \leq 0$. Since $\max \left\{0, \alpha_{0}\right\} \leq \alpha<\alpha_{1}$, by Lemma 2.3.8 (3), $\Delta_{\alpha}^{\prime \prime}(\alpha, 0) \geq 0$. 
By Lemma 2.3.8 (2),

$$
\Delta_{\alpha}^{\prime}(\alpha, 0) \geq \Delta_{\alpha}^{\prime}(0,0)>0 \quad \text { for } \alpha \in\left[\max \left\{0, \alpha_{0}\right\}, \alpha_{1}\right)
$$

This, together with Lemma 2.3.8 (1), implies

$$
\Delta(\alpha, 0)>\Delta(0,0) \geq 0 \quad \text { for } \alpha \in\left[\max \left\{0, \alpha_{0}\right\}, \alpha_{1}\right)
$$

By the continuity of $u^{*}, v^{*}$ and $\Delta_{\alpha}(\alpha, \eta)$ at $\eta$ and (2.3.30), for $\beta>5 / 8$,

$$
\max \left\{\frac{4 \beta^{2}}{1+4 \beta}, \frac{\beta}{2 \sqrt{2 \beta-1}}\right\} \leq \gamma<\beta \quad \text { and } \max \left\{0, \alpha_{0}\right\} \leq \alpha<\alpha_{1}
$$

there exists $\eta_{1}^{*} \in\left(0, \eta_{0}\right)$ such that

$$
\Delta(\alpha, 0)>0 \text { for } \eta \in\left[0, \eta_{1}^{*}\right]
$$

By (2.3.20) and (2.3.22), $\left|A\left(u^{*}, v^{*}\right)\right|>0$ and $\operatorname{tr}\left(A\left(u^{*}, v^{*}\right)\right)<0$. The result follows from Lemma 1.2.1 $(i i)$.

(2) If $\beta \geq 1, \frac{\beta}{2 \sqrt{2 \beta-1}} \leq \gamma \leq \frac{\beta}{2}$ and $0 \leq \alpha<\alpha_{0}$, then by (2.3.30), there exists $\eta^{*} \in\left(0, \eta_{1}\right) \subset\left(0, \eta_{0}\right)$ such that

$$
\Delta(\alpha, 0)>0 \text { for } \eta \in\left[0, \eta_{2}^{*}\right]
$$

By (2.3.20) and (2.3.22), $\left|A\left(u^{*}, v^{*}\right)\right|>0$ and $\operatorname{tr}\left(A\left(u^{*}, v^{*}\right)\right)<0$. The result follows from Lemma 1.2.1 $(i i)$.

Lemma 2.3.11. (1) $\frac{\beta}{2 \sqrt{2 \beta-1}} \leq \frac{\beta}{2}<\frac{4 \beta^{2}}{1+4 \beta}$ for $\beta \geq 1$.

(2) If $\beta \geq 5 / 8$, then there exists a unique $\beta^{*} \in(5 / 8,1)$ such that $\frac{4 \beta^{2}}{1+4 \beta} \leq$ $\frac{\beta}{2 \sqrt{2 \beta-1}}$ for $\beta \in\left(5 / 8, \beta^{*}\right)$ and $\frac{4 \beta^{2}}{1+4 \beta}>\frac{\beta}{2 \sqrt{2 \beta-1}}$ for $\beta>\beta^{*}$. 
Proof. The proof of (1) is straightforward. We prove (2). For $\beta \geq 5 / 8$, let $\xi=\frac{\beta}{2 \sqrt{2 \beta-1}}-\frac{4 \beta^{2}}{1+4 \beta}$. Then we have

$$
\begin{aligned}
\xi & =\frac{\beta(1+4 \beta-8 \beta \sqrt{2 \beta-1})}{2(1+4 \beta) \sqrt{2 \beta-1}}=\frac{\beta\left[(1+4 \beta)^{2}-[8 \beta \sqrt{2 \beta-1})\right]^{2}}{2(1+4 \beta)[1+4 \beta+8 \beta \sqrt{2 \beta-1})] \sqrt{2 \beta-1}} \\
& =\frac{\beta h(\beta)}{2(1+4 \beta)[1+4 \beta+8 \beta \sqrt{2 \beta-1})] \sqrt{2 \beta-1}},
\end{aligned}
$$

where

$$
h(\beta)=128 \beta^{3}-80 \beta^{2}-8 \beta-1 \quad \text { for } \beta>5 / 8 .
$$

Since

$h^{\prime}(\beta)=8\left(48 \beta^{2}-20 \beta-1\right)=384\left(\beta+\frac{2 \sqrt{33}-5}{24}\right)\left(\beta-\frac{2 \sqrt{33}+5}{24}\right)>0 \quad$ for $\beta>5 / 8$,

$h$ is strictly increasing on $(5 / 8, \infty)$. Since $h(5 / 8)<0$ and $h(1)>$, it follows that there exists a unique $\beta^{*} \in(5 / 8,1)$ such that $h\left(\beta^{*}\right)=0, h(\beta)<0$ for $\beta \in\left(5 / 8, \beta^{*}\right)$ and $h(\beta)>0$ for $\beta \in\left(\beta^{*}, \infty\right)$. This, together with (2.3.31), shows that the result (2) holds.

It is easy to verify that

$$
\max \left\{\frac{4 \beta^{2}}{1+4 \beta}, \frac{\beta}{2 \sqrt{2 \beta-1}}\right\}<\beta \quad \text { for } \beta>5 / 8
$$

and

$$
\frac{2 \beta-1}{4}<\frac{4 \beta^{2}}{1+4 \beta} \quad \text { for } \beta>0
$$

Let

$$
\alpha_{2}(\beta, \gamma)=\frac{1}{2}-\frac{\sqrt{\omega(\beta, \gamma)}}{\sqrt{12} \beta} \quad \text { and } \alpha_{3}(\beta, \gamma)=\frac{1}{2}+\frac{\sqrt{\omega(\beta, \gamma)}}{\sqrt{12} \beta}
$$


Lemma 2.3.12. Assume that one of the following conditions holds.

(i) $0<\beta \leq 1 / 4$ and $\frac{\beta}{\sqrt{2(1+\beta)}} \leq \gamma<\frac{\beta}{\sqrt{2(1-2 \beta)}}$.

(ii) $1 / 4<\beta \leq 5 / 8$ and $\frac{\beta}{\sqrt{2(1+\beta)}} \leq \gamma<\beta$.

(iii) $\beta>5 / 8$ and $\frac{\beta}{\sqrt{2(1+\beta)}} \leq \gamma<\frac{\beta}{2 \sqrt{2 \beta-1}}$.

Then $\alpha_{0} \leq \alpha_{2}(\beta, \gamma)$.

Proof. By Lemma 2.3.9 (1) we see that under each of conditions $(i),(i i)$ and (iii) we have $\omega(\beta, \gamma)>0$. If either $1 / 2 \leq \beta \leq 5 / 8$ and $0<\gamma<\beta$ or $($ iii $)$ holds, then

$$
\begin{aligned}
\alpha_{2}(\beta, \gamma) & =\frac{1}{2}-\frac{\sqrt{\omega(\beta, \gamma)}}{2 \sqrt{3} \beta}=\frac{\sqrt{3} \beta-\sqrt{\omega(\beta, \gamma)}}{2 \sqrt{3} \beta}=\frac{3 \beta^{2}-\left(\beta^{2}-8 \beta \gamma^{2}+4 \gamma^{2}\right)}{2 \sqrt{3} \beta(\sqrt{3} \beta+\sqrt{\omega(\beta, \gamma)})} \\
& =\frac{\beta^{2}+2(2 \beta-1) \gamma^{2}}{\sqrt{3} \beta(\sqrt{3} \beta+\sqrt{\omega(\beta, \gamma)})} .
\end{aligned}
$$

and $\alpha_{2}(\beta, \gamma)>0$. If either $(i)$ or $1 / 4<\beta<1 / 2$ and $0<\gamma<\beta$ holds, then by (2.3.33), we obtain

$$
\alpha_{2}(\beta, \gamma)=\frac{(1-2 \beta)\left[\frac{\beta^{2}}{2(1-2 \beta)}-\gamma^{2}\right]}{\sqrt{3} \beta(\sqrt{3} \beta+\sqrt{\omega(\beta, \gamma)})}
$$

Note that if $1 / 4<\beta<1 / 2$, then $\beta<\frac{\beta}{\sqrt{2(1-2 \beta)}}$. Hence, by (2.3.34) we see that under either $(i)$ or $1 / 4<\beta<1 / 2$ and $0<\gamma<\beta$, we have $\alpha_{2}(\beta, \gamma)>0$. 
Since $\gamma \geq \frac{\beta}{2 \sqrt{2(\beta+1)}}$, we have

$$
\begin{aligned}
\alpha_{2}(\beta, \gamma)-\alpha_{0} & =\frac{\gamma}{\beta}-\frac{\sqrt{\omega(\beta, \gamma)}}{2 \sqrt{3} \beta}=\frac{2 \sqrt{3} \gamma-\sqrt{\omega(\beta, \gamma)}}{2 \sqrt{3} \beta}=\frac{12 \gamma^{2}-\omega(\beta, \gamma)}{2 \sqrt{3} \beta(2 \sqrt{3} \gamma+\sqrt{\omega(\beta, \gamma)})} \\
& =\frac{8(\beta+1) \gamma^{2}-\beta^{2}}{2 \sqrt{3} \beta(2 \sqrt{3} \gamma+\sqrt{\omega(\beta, \gamma)})} \\
& =\frac{8(\beta+1)}{2 \sqrt{3} \beta(2 \sqrt{3} \gamma+\sqrt{\omega(\beta, \gamma)})}\left(\gamma^{2}-\frac{\beta^{2}}{8(\beta+1)}\right) \\
& =\frac{8(\beta+1)}{2 \sqrt{3} \beta(2 \sqrt{3} \gamma+\sqrt{\omega(\beta, \gamma)})}\left(\gamma+\frac{\beta}{2 \sqrt{2(\beta+1)}}\right)\left(\gamma-\frac{\beta}{2 \sqrt{2(\beta+1)}}\right) \\
& \geq 0
\end{aligned}
$$

It follows that $\alpha_{0} \leq \alpha_{2}(\beta, \gamma)$.

Notation: Let $\varrho=\max \left\{\frac{\beta}{\sqrt{2(1+\beta)}}, \frac{4 \beta^{2}}{1+4 \beta}\right\}$. It is easy to verify that $0<$ $\beta<1 / 2$, then $\frac{4 \beta^{2}}{1+4 \beta}<\frac{\beta}{\sqrt{2(1-2 \beta)}}$ and by Lemma 2.3.11, $\frac{4 \beta^{2}}{1+4 \beta}<\frac{\beta}{2 \sqrt{2 \beta-1}}$ for $\beta \in\left(5 / 8, \beta^{*}\right)$.

Theorem 2.3.13. Assume that one of the following conditions holds.

(1) $0<\beta \leq 1 / 4$ and $\varrho \leq \gamma<\frac{\beta}{\sqrt{2(1-2 \beta)}}$.

(2) $1 / 4<\beta \leq 5 / 8$ and $\varrho \leq \gamma<\beta$.

(3) $5 / 8<\beta<\beta^{*}$ and $\varrho \leq \gamma<\frac{\beta}{2 \sqrt{2 \beta-1}}$.

Then for each $\alpha \in\left[\max \left\{0, \alpha_{0}\right\}, \min \left\{\alpha_{1}, \alpha_{2}\right\}\right]$, there exists $\eta^{*} \in\left(0, \eta_{0}\right)$ such that $\left(u^{*}, v^{*}\right)$ is a stable node of $(1.4 .3)$ for each $\eta \in\left[0, \eta^{*}\right)$.

Proof. Let $\alpha^{*}=\min \left\{\alpha_{1}, \alpha_{2}\right\}$. By Lemma 2.3.12, we see that $\max \left\{0, \alpha_{0}\right\} \leq$ $\alpha^{*}$ and it makes sense to choose $\alpha$ to satisfy $\max \left\{0, \alpha_{0}\right\} \leq \alpha \leq \alpha^{*}$. By Lemma 2.3.8 (1), (2), (2.3.32) and Lemma 2.3.9 (1), we see that under each of the conditions $(i),(i i)$ and $(i i i), \Delta(0,0) \geq 0, \Delta_{\alpha}^{\prime}(0,0)>0$ and $\omega(\beta, \gamma)>0$. 
Note that each of the conditions $(i),(i i)$ and $(i i i)$ implies $\gamma<\beta$. Hence, we have for $0 \leq \alpha \leq \alpha^{*}$,

$$
\Delta^{\prime \prime}(\alpha, 0)=\frac{12 \beta^{2}}{\gamma^{2}}\left[\left(\alpha-\frac{1}{2}\right)^{2}-\frac{\omega(\beta, \gamma)}{12 \beta^{2}}\right]=\frac{12 \beta^{2}}{\gamma^{2}}\left(\alpha-\alpha_{2}\right)\left(\alpha-\alpha_{3}\right) \geq 0
$$

This implies that the function $\Delta^{\prime}(\cdot, 0)$ is increasing on $\left[0, \alpha^{*}\right]$ and

$$
\Delta_{\alpha}^{\prime}(\alpha, 0) \geq \Delta_{\alpha}^{\prime}(0,0)>0 \quad \text { for } \alpha \in\left[0, \alpha^{*}\right]
$$

and by Lemma 2.3 .8 (1), we have

$$
\Delta(\alpha, 0)>\Delta(0,0) \geq 0 \quad \text { for } \alpha \in\left[0, \alpha^{*}\right]
$$

Hence, for each $\alpha \in\left[\max \left\{0, \alpha_{0}\right\}, \alpha^{*}\right]$, we have $\Delta(\alpha, 0)>0$. By the continuity of $u^{*}, v^{*}$ and $\Delta(\alpha, \eta)$ at $\eta$, there exists $\eta_{1}^{*} \in\left(0, \eta_{0}\right)$ such that

$$
\Delta(\alpha, 0)>0 \text { for } \eta \in\left[0, \eta_{1}^{*}\right]
$$

By Theorem 2.3.6 (1) and (2) $(i),\left|A\left(u^{*}, v^{*}\right)\right|>0$ and $\operatorname{tr}\left(A\left(u^{*}, v^{*}\right)\right)<0$. The result follows from Lemma 1.2.1 (ii).

\subsection{Discussion}

We exhibit that both prey refuge $\alpha$ and the harvesting rate $\eta$ heavily affect the positive equilibria and the local stability of the model (1.4.3). For example, if the harvesting rate $\eta$ is strictly greater than $1 / 4,(1.4 .3)$ has no positive equilibria, which implies that the mutual extinction of prey and predators occurs and the necessary condition for the two species to coexist is $0 \leq \eta \leq$ 
1/4. We give all the ranges of the parameters under which (1.4.3) has a unique positive boundary equilibrium, two positive boundary equilibria, and a positive interior equilibrium. In particular, to ensure that positive interior equilibrium exists, both prey refuge $\alpha$ and harvesting rate $\eta$ can not be too large, that is, they must satisfy $\alpha \in\left[0, \alpha_{1}\right)$ and $\eta \in\left[0, \eta_{0}\right)$. We prove that when $\eta=1 / 4,(1.4 .3)$ has a unique positive equilibrium $(1 / 2,0)$, which is justified to be a saddle-node or topological saddle. When $0 \leq \eta<1 / 4$, (1.4.3) has two positive boundary equilibria, which may be saddle, unstable node, stable node or saddle-node, depending on the choices of prey refuge $\alpha$ and the harvesting rate $\eta$. In particular, when $\eta=0$, we show that the boundary equilibria $(0,0)$ is a saddle, which is same as the result obtained in [7] by a different approach, while another equilibria $(1,0)$ could be saddle, stable node or saddle-node depending on the choices of prey refuge $\alpha$. However, the equilibria $(1,0)$ was proved in [7] only to be a saddle for arbitrary prey refuge $\alpha$. Our result shows that the result in [7] is not true in some cases, see Corollary 2.3.5. We show that the positive interior equilibrium is locally asymptotically stable and could be a stable node under suitable restrictions on the prey refuge and sufficiently small harvesting rate. The last results of Section 2.3, Theorem 2.3.6 and Theorem 2.3.13, are new. 


\section{Chapter 3}

\section{Numerical Simulations}

In this chapter we provide numerical simulations for the main results obtained in Chapter 2. In particular, we provide evidence that the boundary equilibrium $(1,0)$ can be a stable node or a saddle-node respectively, depending on the values of the parameters. Also, we provide evidence that the interior equilibrium $\left(u^{*}, v^{*}\right)$ can be locally asymptotically stable or a stable node depending on the values of the parameters. In all the examples in this chapter, we consider system (1.4.3) with the parameter $\eta=0$, which corresponds to the following system:

$$
\left\{\begin{array}{l}
\dot{u}=u(1-u)-(u-\alpha) v \\
\dot{v}=\beta(u-\alpha) v-\gamma v
\end{array}\right.
$$

In the following examples, we examine system (3.0.1) under specific parameter values for $\beta, \gamma$ and $\alpha$. The parameters are determined by following the Theorems and Corollaries in Section 2.3, in order to demonstrate the results. 


\section{$3.1 \quad$ Example 1}

When $\gamma=\frac{1}{3}, \beta=1$ and $\alpha=\frac{1}{2}$, system (3.0.1) becomes:

$$
\left\{\begin{array}{l}
\dot{u}=u(1-u)-(u-1 / 2) v \\
\dot{v}=(u-1 / 2) v-v / 3
\end{array}\right.
$$

By Corollary 2.3.5 (1) under the conditions $\gamma=\frac{1}{3}, \beta=1$ and $\alpha=\frac{1}{2}$, we have $(1,0)$ is a saddle of system (3.1.1). Below, Figure 3.1 shows numerical solutions of system (3.1.1). The equilibrium $(1,0)$ is a saddle of (3.1.1) since $\gamma=\frac{1}{3}, \beta=1$ and $\alpha=\frac{1}{2}$.

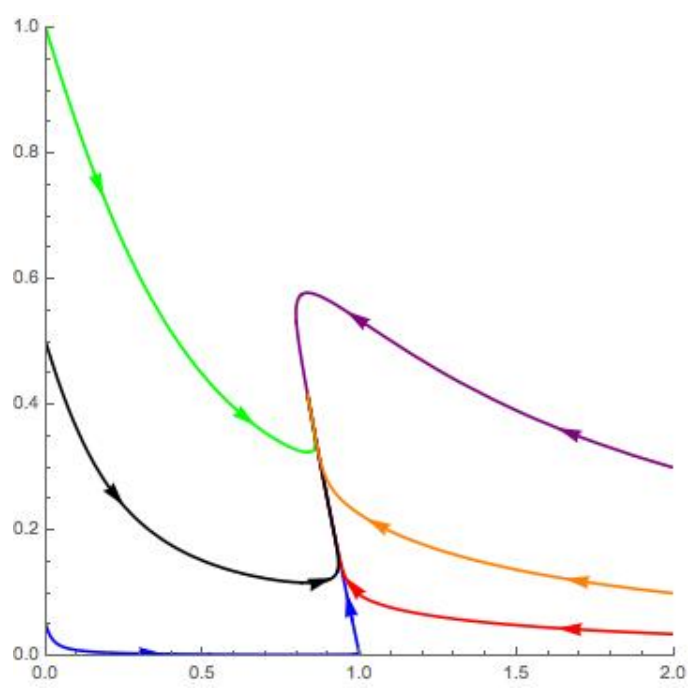

Figure 3.1: Saddle Boundary Equilibrium (Ex. 1) 


\section{$3.2 \quad$ Example 2}

When $\gamma=2, \beta=1$ and $\alpha=\frac{1}{2}$, system (3.0.1) becomes:

$$
\left\{\begin{array}{l}
\dot{u}=u(1-u)-(u-1 / 2) v, \\
\dot{v}=(u-1 / 2) v-2 v .
\end{array}\right.
$$

By Corollary 2.3.5 (2) under the conditions $\gamma=2, \beta=1$ and $\alpha=\frac{1}{2}$, we have $(1,0)$ is a stable node of system (3.2.1). Below, Figure 3.2 shows numerical solutions of system (3.2.1). The equilibrium $(1,0)$ is a stable node of (3.1.1) with $\gamma=2, \beta=1$ and $\alpha=\frac{1}{2}$.

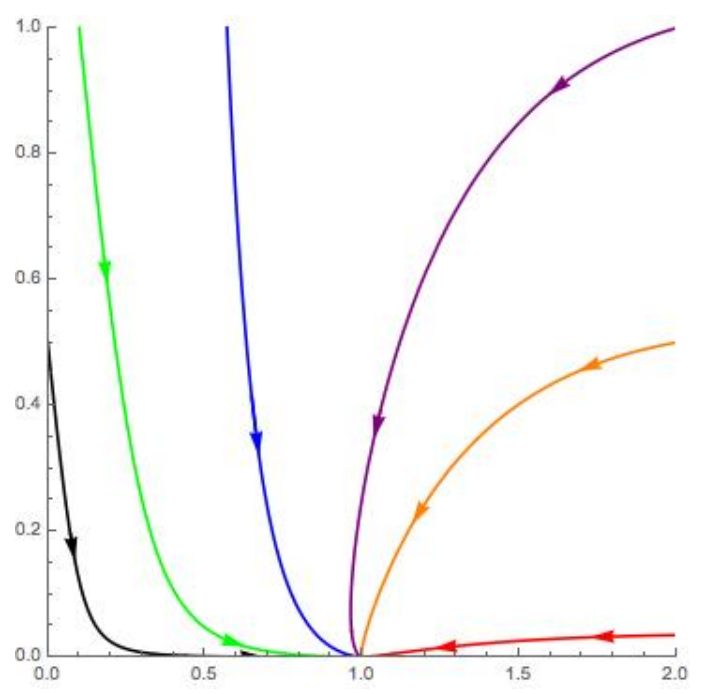

Figure 3.2: Stable Node Boundary Equilibrium (Ex. 2) 


\subsection{Example 3}

When $\gamma=\frac{1}{2}, \beta=1$ and $\alpha=\frac{1}{2}$, system (3.0.1) becomes:

$$
\left\{\begin{array}{l}
\dot{u}=u(1-u)-(u-1 / 2) v \\
\dot{v}=(u-1 / 2) v-v / 2
\end{array}\right.
$$

By Corollary 2.3.5 (3) under the conditions $\gamma=2, \beta=1$ and $\alpha=\frac{1}{2}$, we have $(1,0)$ is a saddle-node of system (3.3.1). Below, Figure 3.3 shows numerical solutions of system (3.3.1). The equilibrium $(1,0)$ is a saddle-node of (3.3.1) with $\gamma=\frac{1}{2}, \beta=1$ and $\alpha=\frac{1}{2}$.

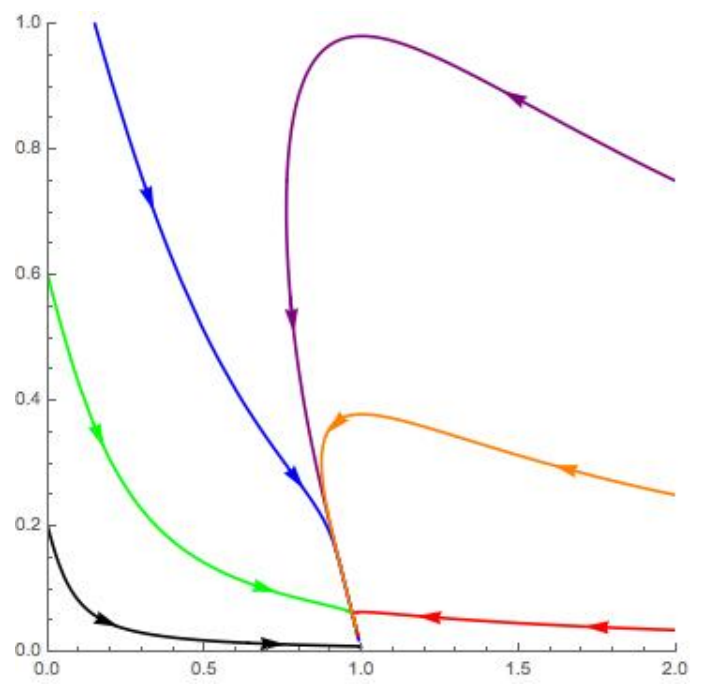

Figure 3.3: Saddle-node Boundary Equilibrium (Ex 3) 


\section{$3.4 \quad$ Example 4}

When $\gamma=\frac{1}{2}, \beta=2$ and $\alpha=\frac{1}{8}$, system (3.0.1) becomes:

$$
\left\{\begin{array}{l}
\dot{u}=u(1-u)-(u-1 / 8) v \\
\dot{v}=2(u-1 / 8) v-v / 2
\end{array}\right.
$$

By Theorem 2.3.6 (1) - (i) under the conditions $\gamma=\frac{1}{2}, \beta=2$ and $\alpha=\frac{1}{8}$, we have $\left(u^{*}, v^{*}\right)=\left(\frac{3}{8}, \frac{15}{16}\right)$ is a locally asymptotically stable equilibrium of system (3.4.1). Below, Figure 3.4 shows numerical solutions of system (3.4.1). The equilibrium $\left(u^{*}, v^{*}\right)=\left(\frac{3}{8}, \frac{15}{16}\right)$ is a locally asymptotically stable equilibrium of (3.4.1) with $\gamma=\frac{1}{2}, \beta=2$ and $\alpha=\frac{1}{8}$.

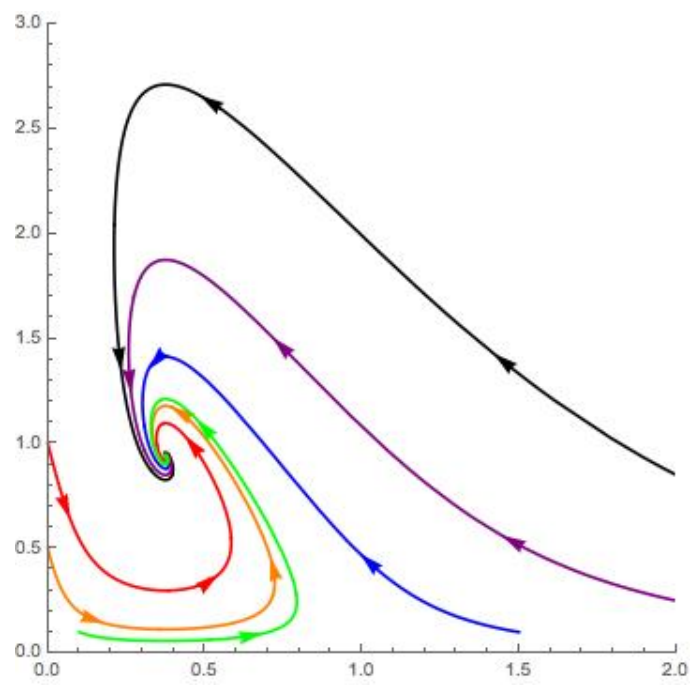

Figure 3.4: Locally Asymptotically Stable Positive Interior Equilibrium (Ex. 4) 


\subsection{Example 5}

When $\gamma=1, \beta=2$ and $\alpha=\frac{1}{4}$, system (3.0.1) becomes:

$$
\left\{\begin{array}{l}
\dot{u}=u(1-u)-(u-1 / 4) v \\
\dot{v}=2(u-1 / 4) v-v
\end{array}\right.
$$

By Theorem 2.3.6 (1), (ii) under the conditions $\gamma=1, \beta=2$ and $\alpha=\frac{1}{4}$, we have $\left(u^{*}, v^{*}\right)=\left(\frac{3}{4}, \frac{3}{8}\right)$ is a locally asymptotically stable equilibrium of system (3.5.1). Below, Figure 3.5 shows numerical solutions of system (3.5.1). The equilibrium $\left(u^{*}, v^{*}\right)=\left(\frac{3}{4}, \frac{3}{8}\right)$ is a locally asymptotically stable equilibrium of (3.0.1) with $\gamma=1, \beta=2$ and $\alpha=\frac{1}{4}$. with $\gamma=\frac{1}{2}, \beta=2$ and $\alpha=\frac{1}{8}$.

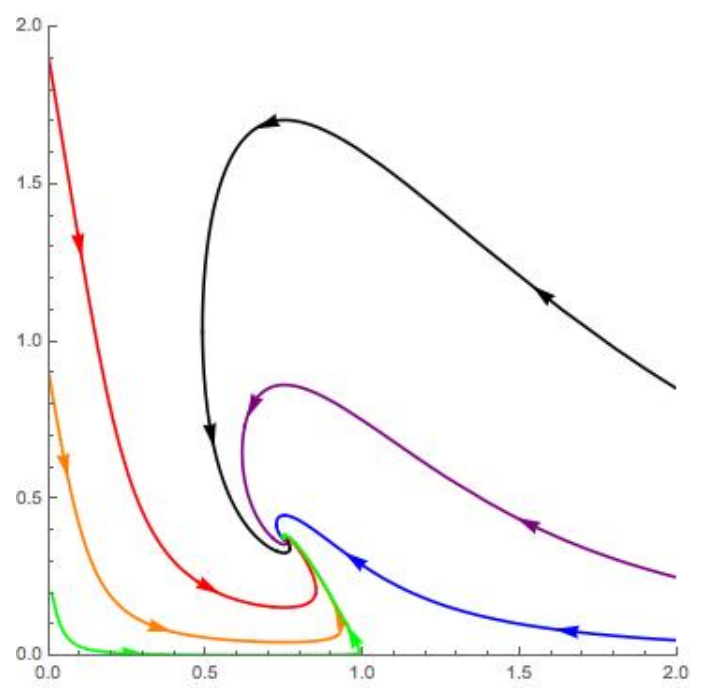

Figure 3.5: Locally Asymptotically Stable Positive Interior Equilibrium (Ex. 5) 


\subsection{Example 6}

When $\gamma=\frac{1}{8}, \beta=\frac{1}{4}$ and $\alpha=\frac{1}{10}$, system (3.0.1) becomes:

$$
\left\{\begin{array}{l}
\dot{u}=u(1-u)-(u-1 / 10) v \\
\dot{v}=\frac{1}{4}(u-1 / 10) v-v / 8
\end{array}\right.
$$

By Theorem 2.3.13 (1) under the conditions $\gamma=\frac{1}{8}, \beta=\frac{1}{4}$ and $\alpha=\frac{1}{10}$, we have $\left(u^{*}, v^{*}\right)=\left(\frac{3}{5}, \frac{12}{25}\right)$ is a stable node of system (3.6.1). Below, Figure 3.6 shows numerical solutions of system (3.6.1). The equilibrium $\left(u^{*}, v^{*}\right)=$ $\left(\frac{3}{5}, \frac{12}{25}\right)$ is a stable node of (3.6.1) with $\gamma=\frac{1}{8}, \beta=\frac{1}{4}$ and $\alpha=\frac{1}{10}$

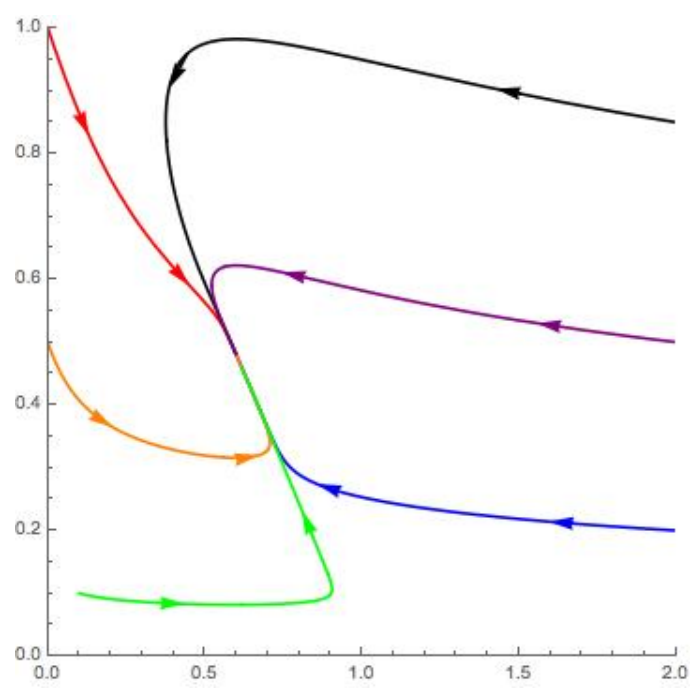

Figure 3.6: Stable Node Positive Interior Equilibrium (Ex. 6) 


\section{7 $\quad$ Example 7}

When $\gamma=\frac{7}{18}, \beta=\frac{1}{2}$ and $\alpha=0$, sy stem (3.0.1) becomes:

$$
\left\{\begin{array}{l}
\dot{u}=u(1-u)-u v \\
\dot{v}=\frac{1}{2} u v-\frac{7}{18} v
\end{array}\right.
$$

By Theorem 2.3.13 (2) under the conditions $\gamma=\frac{7}{18}, \beta=\frac{1}{2}$ and $\alpha=0$, we have $\left(u^{*}, v^{*}\right)=\left(\frac{7}{9}, \frac{54}{343}\right)$ is a stable node of system (3.7.1). Below, Figure 3.7 shows numerical solutions of system (3.6.1). The equilibrium $\left(u^{*}, v^{*}\right)=\left(\frac{7}{9}, \frac{54}{343}\right)$ is a stable node of (3.7.1) with $\gamma=\frac{7}{18}, \beta=\frac{1}{2}$ and $\alpha=0$.

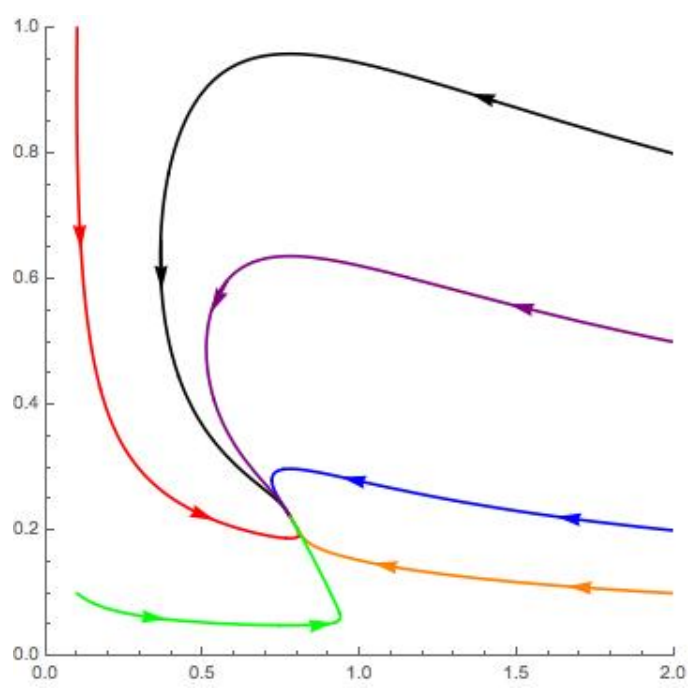

Figure 3.7: Stable Node Positive Interior Equilibrium (Ex. 7) 


\section{Bibliography}

[1] A. A. Andronov, E. A. Leontovich, I. I. Gordon and A. G. Maier, Qualitative Theory of Second-Order Dynamical Systems, John Wiley and Sons, New York, 1973.

[2] L. Allen, An Introduction to Mathematical Biology, Pearson Prentice Hall, 2007

[3] J. R. Beddington, Mutual interference between parasites or predators and its effect on searching efficiency, J. Animal Ecol. 44(1975), 331-340.

[4] P. A. Braza, The bifurcation structure of the Holling-Tanner model for predator-prey interaction using two-timing, SIAM J. Appl. Math. 63(2003), 889-904.

[5] R. S. Cantrell and C. Cosner, On the dynamics of predator-prey models with the Beddington-DeAngelis functional response, J. Math. Anal. Appl. 257(2001), 206-222.

[6] F. D. Chen, Z. Z. Ma and H. Y. Zhang, Global asymptotical stability of the positive equilibrium of LotkaVolterra preypredator model incor- 
porating a constant number of prey refuge, Nonlinear Anal. Real World Appl. 13(2012)(6), 2790-2793.

[7] L. J. Chen, F. D. Chen and Y. Q. Wang, Influence of predator mutual interference and prey refuge on LotkaVolterra predatorprey dynamics, Commun. Nonlinear Sci. Numer. Simulat. 18 (2013), 3174-3180.

[8] L. J. Chen, F. D. Chen and L. J. Chen, Qualitative analysis of a predator-prey model with Holling type II functional response incorporating a constant prey refuge, Nonlinear Anal. Real World Appl. 11 (2010), 246-252.

[9] C. W. Clark, "Mathmatics Bioeconomics, The Optimal Management of Renewable Resources," Second edition, Pure and Applied Mathematics (New York), A Wiley-Interscience Publication, John Wiley \& Sons, Inc., New York, 1990.

[10] D. L. DeAngelis, R. A. Goldstein and R. V. O'Neil, A model for trophic interaction, Ecology, 56(1975), 881-892.

[11] T. D. Doan, C. R. Zhu and K. Q. Lan, Phase plane analysis of the susceptible-infected- removed- susceptible (SIRS) epidemic models with nonlinear incidence rates, J. Nonlinear Funct. Anal. 2016 (2016), Article ID 30 .

[12] E. González-Olivares and R. Ramos-Jiliberto, Dynamic consequences of prey refuges in a simple model system: more prey, fewer predators and enhanced stability, Ecol. Model. 166 (2003), 135-146. 
[13] C. M. Heggerud and K. Q. Lan, Local stability analysis of ratiodependent predator-prey models with predator harvesting rates, Appl. Math. Comput., 270(2015), 349-357.

[14] C. S. Holling, The functional response of predator to prey density and its role in mimicry and population regulation, Mem. Ent. Soc. Can., 45(1965), 1-60.

[15] S. B. Hsu and T. W. Huang, Global stability for a class of predator-prey system, SIAM J. Appl. Math. 55(1995), 763-783.

[16] M. Hoti, X. Huo and K.Q. Lan, Stability and phase portraits of susceptible-infective-removed epidemic models with vertical transmissions and linear treatment rates, Electron. J. Differential Equations, Vol. 2017 (2017), No. 306, 1-17.

[17] S. B. Hsu, T. W. Hwang and Y. Kuang, Global analysis of the MichaelisMenten type ratio-dependent predator-prey system, J. Math. Biol. 42(2001), 489-506.

[18] L. L. Ji and C. Q. Wu, Qualitative analysis of a predatorprey model with constant-rate prey harvesting incorporating a constant prey refuge, Nonlinear Anal. Real World Appl. 11 (2010), 2285-2295.

[19] Y. Kuang and E. Beretta, Global qualitative analysis of a ratiodependent predator-prey system, J. Math. Biol. 36(1998), 389-406. 
[20] K. Q. Lan and C. R. Zhu, Phase portraits of predator-prey systems with harvesting rates, Discrete Contin. Dyn. Syst. Ser. A. 32(2012)(3), 901-933.

[21] K. Q. Lan and C. R. Zhu, Phase portraits, Hopf bifurcations and limit cycles of the Holling-Tanner models for predator-prey interactions, Nonlinear Anal. Real World Appl. 12(2011), 1961-1973.

[22] G. P. Luo, K. Q. Lan and C. R. Zhu, Bifurcations of ratio-dependent predator-prey Holling type III systems with harvesting rates, J. Nonlinear Funct. Anal. 2014 (2014), Article ID 16.

[23] G. P. Luo, C. R. Zhu and K. Q. Lan, Dynamics of an SIR epidemic model with horizontal and vertical transmissions and constant treatment rates, J. Appl. Anal. Comput. 7 (2017)(3), 957-976.

[24] Z. H. Ma, The research of predatorprey models incorporating prey refuges, Ph.D. Thesis, Lanzhou University, P. R. China, 2010.

[25] Z. Z. Ma, F. D. Chen, C. Q. Wu and W. L. Chen, Dynamic behaviors of a LotkaVolterra predatorprey model incorporating a prey refuge and predator mutual interference, Appl. Math. Comput. 219 (2013), 79457953.

[26] R. M. May, Stability and complexity in model ecosystems, Princeton: Prinseton University Press, 1973. 
[27] L. Perko, Differential Equations and Dynamical Systems, SpringerVerlag, New York, 1996.

[28] E. Sáez and E. González-Olivares, Dynamics of a predator-prey model, SIAM J. Appl. Math. 59(1999), 18671878.

[29] G. Seo and G. S. K. Wolkowicz, Existence of multiple limit cycles in a predator-prey model with $\arctan (a x)$ as functional response, Commun. Math. Anal. 1 (2015), 64-68.

[30] G. Seo and M. Kot, A comparison of two predatorprey models with Hollings type I functional response, Math. Biosci. 12 (2008), 161-179.

[31] G. Seo and D. L. DeAngelis, A predatorprey model with a Holling type I functional response including a predator mutual interference, J. Nonlinear Sci. 21 (2011), 811-833.

[32] J. T. Tanner, The stability and the intrinsic growth rates of prey and predator populations, Ecology 56(1975), 855-867.

[33] D. J. Wollkind and J. A. Logan, Temperature-dependent predator-prey mite ecosystem on apple tree foliage, J. Math. Biol. 6(1978), 265-283.

[34] D. J. Wollkind, J. B. Collings and J. A. Logan, Metastability in a temperature-dependent model system for predator-prey mite outbreak interactions on fruit flies, Bull. Math. Biol. 50(1988), 379-409. 
[35] D. Xiao and L. S. Jennings, Bifurcations of a ratio-dependent predatorprey system with constant rate harvesting, SIAM J. Appl. Math. 65(2005), 737-753.

[36] C. R. Zhu and K. Q. Lan, Phase portraits, Hopf bifurcations and limit cycles of Leslie-Gower predator-prey systems with harvesting rates, Discrete Contin. Dyn. Syst. Ser. B 14(2010), 289-306. 


\section{Index}

boundary, iii, 1, 2, 7, 18, 21, 22, $44-48$

carrying capacity, 7

differential equations, 2, 4, 6

dynamical system, 1, 2

equilibria, iii, 7, 12, 13, 18-21, 43, 44

equilibrium, iii, 2-7, 13, 18-22, 26, $29,33,36,44-52$

growth rate, 7

harvesting, iii, 6, 7, 16, 19, 29, 36, 43,44

locally asymptotically stable, iii, $4,5,34,36,44,45,49,50$ node, 4

parameters, iii, 8, 9, 12, 13, 16, $18,36,44,45$

positive interior equilibrium, 7 , $33,44,49,51,52$

predator-prey, iii, 6,7

refuge, $7,16,33,36,43,44$

saddle, 4-6, 22, 26, 29, 30, 33, 44, 46

saddle-node, 5, 22, 23, 26, 29, 30, $33,44,45,48$

solution, 1, 15-19

solutions, 4, 8, 13, 15-17, 19, 46-52

stability, iii, 2, 4, 6, 18, 21, 43

stable, iii, 4, 5, 7, 29, 30, 33, 34, 
$36,38,42,44,45,47$,

$49-52$

system, 1-5, 7-10, 12-16, 18, 19,
$21,23,24,29,33,45-52$

systems, iii, 2, 4, 6

unstable, iii, 4, 5, 26, 34, 44 